\title{
Attitude Quantifier Based Possibility Distribution Generation Method for Hesitant Fuzzy Linguistic Group Decision Making
}

\author{
Jingjing $\mathrm{Hao}^{\mathrm{a}, \mathrm{b}}$, Francisco Chiclana $\mathrm{a}^{\mathrm{b}, \mathrm{c}^{*}}$ \\ ${ }^{a}$ College of Economics and Management, Zhejiang Normal University, Jinhua, Zhejiang, China \\ ${ }^{b}$ Institute of Artificial Intelligence, Faculty of Technology, De Montfort University, Leicester, UK \\ ${ }^{c}$ Andalusian Research Institute on Data Science and Computational Intelligence, University of Granada, Spain.
}

\begin{abstract}
The possibility distribution-based approach is one of the powerful tools available to manage hesitant fuzzy linguistic term set (HFLTS) information. However, existing possibility distribution studies have not considered the experts' satisfied preference for HFLTSs in the process of generating the possibility distribution. This paper aims at filling this research gap. To achieve this goal, a novel possibility distribution generation method based on the concept of linguistic quantifier is proposed. This is accomplished by defining a new attitude linguistic quantifier, which is supported with theoretical results to analyze the relationship between the proposed attitude linguistic quantifier with the original linguistic quantifier, attitude indices and the expected linguistic term. The new possibility distribution generation method is proved to be (1) more general than the two main existing approaches, which are particular cases for specific linguistic quantifiers; and (2) useful to implement the concept of soft majority in the resolution process of the decision making situation. Additionally, a new two stages feedback mechanism of attitude adjustment and assessment adjustment is devised to guarantee the convergence of the consensus reaching process. Finally, a framework of group decision making with HFLTSs information is presented and an illustrative example is conducted to verify the proposed method.
\end{abstract}

Keywords: Hesitant fuzzy linguistic term set, possibility distribution, attitude index, feedback mechanism, consensus reaching process.

\section{Introduction}

In group decision making problems, experts with different backgrounds, cognitive styles and expertise, assess criteria or alternatives based on their preferences and derive a solution by means of an appropriate decision model. However, when experts' assessments are rather different, the obtained collective assessment might not be acceptable to all experts in the group. In such cases, an effective consensus model would be necessary to bring closer the experts' preferences in an attempt to derive an acceptable group decision. Many scholars have developed a large number of consensus methods from different perspectives: consensus measure for various types of preferences [6,7,42]; feedback mechanism [30]; optimization-based consensus model $[45,46]$; consensus model with preference information [49,50]; dynamic consensus process model [21] and consensus model for large scale group decision making [15,18]. Herrera-Viedma et al. in [12] presented a clear overview of recent achievements concerning 'soft consensus models' in a fuzzy environment, while Zhang et al. carried out a comparative study on different consensus reaching approaches and proposed comparison criteria to evaluate the efficiency of consensus reaching processes [48]. As multiple experts are involved in the whole consensus reaching process, the feedback mechanism of the consensus model is inevitably affected by different cognitive styles of experts, which is rarely considered in current research literature on this topic. An objective of the present paper is to develop a novel feedback mechanism that implements different attitudes of experts in a fuzzy environment.

In group decision making problems, some researchers utilized various fuzzy number such as intuitionistic fuzzy number or hesitant fuzzy number to evaluate the alternatives[9-11]. However, experts may prefer subjective words or sentences to numbers as more intuitive and convenient to assess the options or alternatives. Zadeh [43] introduced the concept of linguistic variable to describe variables in the context of natural language frameworks, which has been taken forward by many researchers to propose a number of different methods towards the development of the linguistic computational model: semantic based model [8]; ordinal linguistic model [35,37]; linguistic 2-tuple representation model [13]; virtual linguistic model [32].

Considering the constraints of complex circumstances, uncertain information and diverse knowledge

${ }^{*}$ Corresponding author

E-mail addresses: redearjing@126.com (J. J. Hao), chiclana@dmu.ac.uk (F. Chiclana) 
backgrounds, experts might believe that the provision of a single linguistic term is not sufficient to represent their opinions or preferences when assessing alternatives and they might be hesitant about several linguistic terms. Although some of the above mentioned linguistic models involve uncertain linguistic expression, they are not efficient enough to express the hesitant intent. Based on the linguistic approach [43] and the concept of hesitant fuzzy set [25], Rodríguez et al. [22] introduced the concept of hesitant fuzzy linguistic term set (HFLTS) to describe the hesitant situation in linguistic decision-making evaluation problems. The concept of HFLTS has attracted many scholars' attention and has been developed in many areas [1,16,17,23,27]. Wang et al. in [29] summarized the current methodologies for hesitant fuzzy linguistic decision-making. The possibility distribution based method is a computational method for HFLTSs that was proposed by $\mathrm{Wu}$ and $\mathrm{Xu}$ [31], who considered that expert's HFLTS assessment value is, in the absence of further information, a discrete set of predefined linguistic terms with equal possibility degrees. Their possibility distribution based method provided a new computational strategy to manage the HFLTSs. By considering experts' different attitudes due to individual differences in cognitive styles, Chen et al. [4] developed new possibility distribution generation methods based on probability density functions (PDFs). Furthermore, Pang et al. [20] introduced a new concept of probabilistic linguistic term sets (PLTSs) to describe the probability distributions of HFTLSs, where the probabilistic information is directly or indirectly given by the decision makers. Chen et al. [3] proposed a similar concept named proportional HFLTSs (PHFLTSs) that includes the proportional information of each generalized linguistic term calculated by counting how many experts support each linguistic expression. Both Pang et al. [20] and Chen et al. [3] recognized different possibility values of linguistic terms in HFLTSs from the group perspective. However, they did not address the issue of how to derive the different possibility values of linguistic terms in HFLTSs from the individual expert's preference. In terms of an individual expert, his/her preference of possibility distribution for HFLTSs is affected by many factors such as expertise, habit, knowledge, experience, attitude etc. Also different experts may have different preferences for the same HFLTSs. Therefore, it is meaningful to explore the influences of experts' preference on the possibility distribution for HFLTSs, which is the main objective that drives the present research study.

Experts' preference should be reflected by the elements of the HFLTS that they are satisfied with. For example, experts might be satisfied with all or most of the elements in the HFLTSs. On the contrary, experts might be satisfied with few or several of the elements in the HFLTSs. In other words, we can describe experts' preference through the proportion of elements in the HFLTSs they are satisfied with. This motivates us to propose the use of the concept of linguistic quantifier, which has been widely applied in computing with word theory, to express the experts' preference for HFLTSs. The linguistic quantifier, developed by Zadeh [44], is represented typically by terms such as most, all, about and at least half. Zadeh divided the family of linguistic quantifier into two classes, absolute and relative, while Yager [34,38] further developed the relative quantifier, of which there are three types: regular increasing monotone (RIM) quantifier; regular decreasing monotone (RDM) quantifier; and regular unimodal (RUM) quantifier. Yager also proposed a RIM linguistic quantifier guided method to obtain the OWA aggregation weights [38]. Considering that the properties imposed to OWA weights coincide with those of possibility degrees, the OWA weighting vector can be viewed as a "kind" of probability distribution [39]. This is exploited here to achieve, as mentioned above, the main objective of the present research study, which is the exploring and developing of a new possibility distribution generation method with linguistic quantifier based OWA weights.

Whether in terms of HFLTSs as an assessment information or linguistic quantifier as the reflection of satisfied preference, they are relevant to the cognitive styles and subjective uncertainty of experts. Attitudes are recognized as a useful element to express the characteristic of subjective preference. Many researchers realized the influences of the attitude in group decision making problems and incorporate experts' attitude into the decision making process $[2,26,40]$. However, they rarely involve the attitude-related hesitant fuzzy linguistic model, which is still a new field that needs to be explored. Although an attitude parameter is considered by Chen et al. [4] in their possibility distribution generation method, this cannot reflect the intuitive satisfied preference for HFLTSs. Different from current studies, the approach put forward in the present paper incorporates attitudes relevant to linguistic quantifier into a hesitant fuzzy linguistic model. The proposed attitude linguistic quantifier and OWA weighting vector approach will allow us to propose a new possibility distribution generation method for HFLTSs and its application in group decision-making problems.

The remainder of this paper is organized as follows. In order to make the paper self-contained, in Section 2 some fundamental concepts and methods are recalled. Section 3 presents the possibility distribution generation methods 
for HFLTSs with linguistic quantifier. In section 4, the attitude linguistic quantifier is defined, and the computational model of HFLTSs with expected linguistic value is developed. Section 5 proposes a novel consensus reaching framework in GDM problems characterized by a new feedback mechanism that combines experts' attitudes. In Section 6, the group decision making framework with HFLTSs information is presented, which is illustrated with an example. Section 7 summarizes the proposed methods presented in the paper.

\section{Preliminaries}

\subsection{Hesitant fuzzy linguistic term sets (HFLTSs) and possibility distribution for HFLTSS}

The concept of hesitant fuzzy linguistic term sets (HFLTSs) was introduced in [22] to describe hesitancy in linguistic decision making evaluation problems.

Definition 1: "Let $S=\left\{s_{0}, s_{1}, \ldots, s_{g}\right\}$ be a linguistic term set, a hesitant fuzzy linguistic term set (HFLTS), denoted as $H_{S}$, is an ordered finite subset of consecutive linguistic terms of $S$."

Based on the linguistic distribution assessment [47], $\mathrm{Wu}$ and $\mathrm{Xu}$ [31] introduced the following possibility distribution method to manage HFLTSs.

Definition 2: "Let $S=\left\{s_{0}, s_{1}, \ldots, s_{g}\right\}$ be a linguistic term set. Let $H_{S}=\left\{s_{L}, s_{L+1}, \ldots, s_{U}\right\}$ be a HFLTS given by an expert with $0 \leq L \leq U \leq g$. The possibility distribution for $H_{S}$ on $S$ is represented by $P=\left\{p_{0}, \ldots, p_{l}, \ldots, p_{g}\right\}$,

$$
p_{l}=\left\{\begin{array}{l}
0, \quad l=0,1, \ldots, L-1 \\
\frac{1}{U-L+1}, \quad l=L, \ldots, U, \\
0, \quad l=U+1, \ldots, g
\end{array}\right.
$$

where $\sum_{l=0}^{g} p_{l}=1$, and $0 \leq p_{l} \leq 1(l=0, \ldots, g)$ denotes the possibility that an alternative has an assessment value $s_{l}$ provided by the expert."

$\mathrm{Wu}$ and $\mathrm{Xu}$ [31] uniform possibility distribution represents a scenario where experts do not provide any further information on the possibility degrees associated to the linguistic terms they use to form their corresponding HFLTSs. Thus, in the presence of additional information such as attitude, expertise and/or experience, the possibility distribution for HFLTSs might not be best modeled using a uniform distribution. In these scenarios, Chen et al. [4] proposed the following new possibility distribution generation method based on probability density functions (PDFs).

Definition 3: "Let $S=\left\{s_{0}, s_{1}, \ldots, s_{g}\right\}$ be a linguistic term set. Let $H_{S}=\left\{s_{L}, s_{L+1}, \ldots, s_{U}\right\}$ be a HFLTS given by an expert with $0 \leq L \leq U \leq g$. The possibility distribution for $H_{S}$ on $S$ generated from the PDF $f(x)$ for a continuous random variable $X$ is represented by $P=\left\{p_{0}, \ldots, p_{l}, \ldots, p_{g}\right\}$, where $p_{l}$ is given by

$$
p_{l}=\left\{\begin{array}{c}
0, \quad l=0,1, \ldots, L-1 \\
\frac{f(l)}{\sum_{k=L}^{U} f(k)}, \quad l=L, \ldots, U . \\
0, \quad l=U+1, \ldots, g
\end{array}\right.
$$

\subsection{Regular Increasing Monotone (RIM) quantifier}

Yager $[38,39]$ developed the relative quantifier and gave a definition of Regular Increasing Monotone (RIM) quantifier as follows.

Definition 4: "A Regular Increasing Monotone (RIM) quantifier is a fuzzy subset of the real line with associated membership function $Q$ verifying the following three properties: $1 . Q(0)=0 ; 2 . Q(1)=1 ; 3 . Q(x) \geq Q(y)$ if $x>$ y." 


\subsection{Linguistic quantifier guided OWA aggregation}

The quantifier guided OWA aggregation weights derivation approach introduced by Yager in [38] is a powerful tool to implement the concept of fuzzy majority $[38,39]$.

Definition 5: "An aggregation operator $F, F: I^{n} \rightarrow I, F\left(a_{1}, \ldots, a_{n}\right)=\sum_{j=1}^{n} w_{j} b_{j}$, of dimension $n$ with an associated weighting vector $W=\left(w_{1}, \ldots, w_{n}\right)$ satisfying $0 \leq w_{j} \leq 1$ and $\sum_{j=1}^{n} w_{j}=1$, in which $b_{j}$ is the $j$-th largest value of the set $\left\{a_{1}, \ldots, a_{n}\right\}$ is called an Ordered Weighted Averaging (OWA) operator."

With a RIM quantifier $Q$, the OWA weighting vector components can be obtained as $w_{j}=Q\left(\frac{j}{n}\right)-Q\left(\frac{j-1}{n}\right)$, and the corresponding OWA operator, $F_{Q}$, is called a linguistic quantifier guided OWA aggregation operator. It is worth noting that the properties imposed to $w_{j}$ coincide with the properties of a possibility distribution, and indeed Yager in [39] suggested that the weighting vector can be viewed as a "kind" of probability distribution. Driven by the similarity between OWA weights and possibility values of linguistic terms, a novel possibility distribution generation method for HFLTSs, based on the linguistic quantifier and OWA aggregation, is proposed in the next section.

\section{Quantifier based possibility distribution generation method for HFLTSs}

As mentioned above, the possibility distribution approach proposed by $\mathrm{Wu}$ and $\mathrm{Xu}$ [31] relates the elements in HFLTSs to possibility degrees that are uniformly distributed. Obviously, in real decision making circumstance where experts have different preferences and attitudes, the use of uniform possibility distribution is not the most appropriate. Consequently, Chen et al. [4] developed a possibility density function based method to produce the possibility degree with embedded experts' attitude. Indeed, their method requires experts to have knowledge about the density function of elements in HFLTSs, which actually increases the burden of experts in the decision process. Therefore, the first objective of the present study is to devise an alternative possibility distribution generation method that produces different possibility degrees and is, simultaneously, easy and convenient for experts to implement.

Following Yager's linguistic quantifier guided OWA approach [38], a new framework for managing HFLTSs is proposed where the linguistic quantifier $Q$ is utilized to indicate the proportion of the linguistic terms satisfying the experts' HFLTS preference. In other words, the role of the linguistic quantifier will facilitate the implementation of the statement " $Q$ linguistic terms in HFLTS are satisfied by the experts' preferences", which is more convenient and understandable than the corresponding statement using a specific crisp value. Therefore, the proposed approach in this study integrates the linguistic quantifier into the process of generating possibility degree to propose a new linguistic quantifier guided possibility distribution method. This is stated in the following new definition:

Definition 6: "Let $S=\left\{s_{0}, s_{1}, \ldots, s_{g}\right\}$ be a linguistic term set and $H_{S}=\left\{s_{L}, s_{L+1}, \ldots, s_{U}\right\}$ be a HFLTS given by an expert with $0 \leq L \leq U \leq g$. The possibility distribution for $H_{S}$ on $S$ generated by means of a RIM linguistic quantifier $Q$ is represented by $P=\left\{p_{0}, \ldots, p_{l}, \ldots, p_{g}\right\}$, where $p_{l}$ denotes the possibility degree associated to the linguistic term $s_{l}$ on $S$

$$
p_{l}=\left\{\begin{array}{c}
0, \quad l=0,1, \ldots, L-1 \\
Q\left(\frac{U-l+1}{U-L+1}\right)-Q\left(\frac{U-l}{U-L+1}\right), \quad l=L, \ldots, U \\
0, \quad l=U+1, \ldots, g
\end{array}\right.
$$

subject to the following conditions: $\sum_{l=0}^{g} p_{l}=1$, and $0 \leq p_{l} \leq 1(l=0, \ldots, g)$."

For the purposes of confirmation of the validity of the above definition, in the following we prove that the conditions for the possibility degrees associated to the linguistic term set $S$ are indeed verified. 
Proposition 1: Let $P=\left\{p_{0}, \ldots, p_{l}, \ldots, p_{g}\right\}$ be the set of linguistic quantifier $Q$ guided possibility degrees associated to the linguistic term set $=\left\{s_{0}, s_{1}, \ldots, s_{g}\right\}$. Then the following conditions are satisfied: $\sum_{l=0}^{g} p_{l}=1$, and $0 \leq p_{l} \leq 1(l=0, \ldots, g)$.

Proof: Firstly, from equation (3) we observe that $\sum_{l=0}^{g} p_{l}$ is a telescopic sum; secondly, applying properties 1 and 2 of defintion 4 we finally derive that $\sum_{l=0}^{g} p_{l}=1$.

$$
\sum_{l=0}^{g} p_{l}=\sum_{l=L}^{U}\left[Q\left(\frac{U-l+1}{U-L+1}\right)-Q\left(\frac{U-l}{U-L+1}\right)\right]=Q\left(\frac{U-L+1}{U-L+1}\right)-Q\left(\frac{U-U}{U-L+1}\right)=Q(1)-Q(0)=1
$$

Property 3 of defintion 4 implies that

$$
0 \leq Q\left(\frac{U-l+1}{U-L+1}\right)-Q\left(\frac{U-l}{U-L+1}\right) \leq 1 .
$$

Consequently, it is $0 \leq p_{l} \leq 1(l=0, \ldots, g)$.

Various types of RIM linguistic quantifiers [46,47] can be applied in definition 6 to create abundant linguistic quantifier guided possibility distributions for HFLTSs. In the following, we consider some special cases of RIM quantifier to explain how our method is implemented.

Example 1: Implementing the average linguistic quantifier [40], $Q(x)=x$, the possibility degrees obtained by definition 6 for $l=L, \ldots, U$ are:

$$
p_{l}=Q\left(\frac{U-l+1}{U-L+1}\right)-Q\left(\frac{U-l}{U-L+1}\right)=\frac{U-l+1}{U-L+1}-\frac{U-l}{U-L+1}=\frac{1}{U-L+1}
$$

It is clear that the obtained linguistic quantifier guided possibility distribution coincides with $\mathrm{Wu}$ and Xu's one [31]. Thus, the proposed approach in this study extends the existing uniformly distributed possibility degrees approach of definition 2 .

Example 2: Consider now the linguistic quantifier modeling the soft majority concept "most" defined in [28] as

$$
Q(x)=\left\{\begin{aligned}
0, & x<0.3 \\
2(x-0.3), & 0.3 \leq x \leq 0.8 \\
1, & x>0.8
\end{aligned}\right.
$$

Equation (7) is illustrated in Fig. 1(a). Suppose $H_{S}=\left\{s_{2}, s_{3}, s_{4}, s_{5}\right\}$ is a HFLTS on $S=\left\{s_{0}, s_{1}, \ldots, s_{6}\right\}$, then the possibility distribution derived would be $P=\{0,0,0.1,0.5,0.4,0,0\}$. The special value of $p_{5}$ corresponding to linguistic term $S_{5}$ being equal to 0 is caused by the characteristic of the linguistic quantifier satisfying the fuzzy majority concept to implement. Alternative linguistic quantifiers, "at least half" and "as many as possible", used to model other types of soft majority are illustrated in Fig. 1(b) and Fig. 1(c).

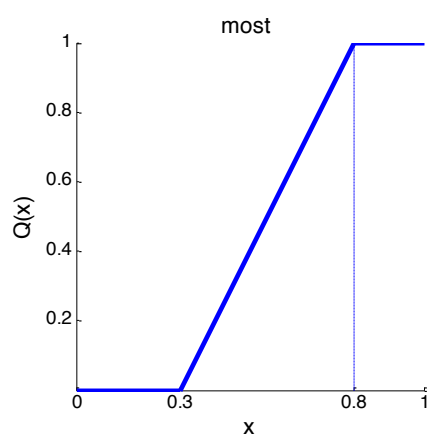

Fig.1(a)

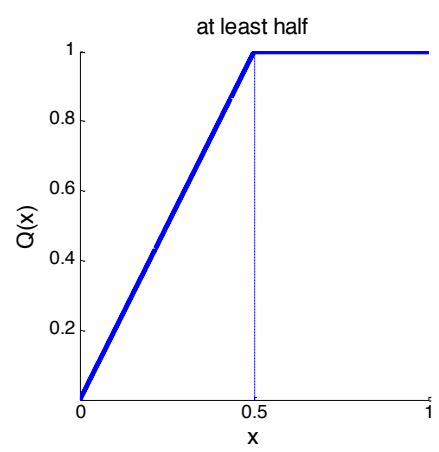

Fig.1(b)

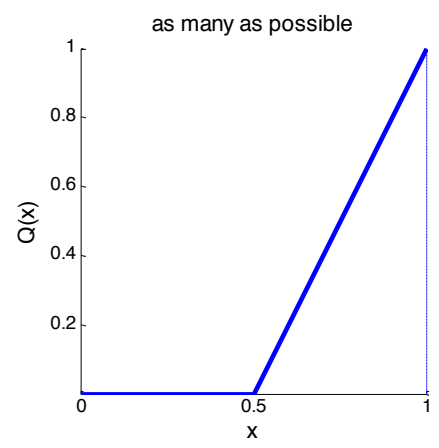

Fig.1(c)

Fig. 1. Linguistic quantifiers 'most', 'at least half' and 'as many as possible'

The new possibility distribution generation method given above in definition 6 can also be applied to non-linear RIM quantifiers, such as the RIM quantifiers with exponential and or power generating function defined in [19], as the following example illustrates. 
Example 3: Consider the below RIM quantifier with exponential generating function [19]

$$
Q(x)=\left\{\begin{array}{cc}
\frac{a^{x}-1}{a-1}, & a>0, a \neq 1 \\
x, & a=1
\end{array} .\right.
$$

Suppose $H_{S}=\left\{s_{2}, s_{3}, s_{4}, s_{5}\right\}$ is a HFLTS on $S=\left\{s_{0}, s_{1}, \ldots, s_{6}\right\}$, and $a=2$. The possibility distribution derived would be $P=\left\{0,0,2-2^{\frac{3}{4}}, 2^{\frac{3}{4}}-2^{\frac{1}{2}}, 2^{\frac{1}{2}}-2^{\frac{1}{4}}, 2^{\frac{1}{4}}-1,0\right\}$. Alternative nonlinear quantifiers with different values of the parameter $a$ are illustrated in Fig. 2.

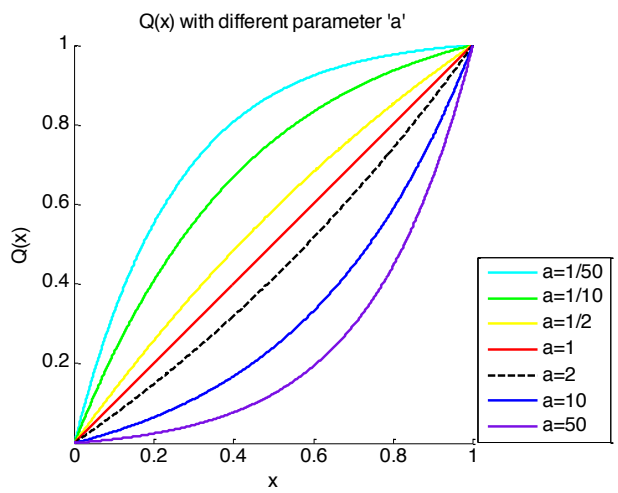

Fig. 2. The shape of $Q(x)$ with different parameter ' $a$ '

The induced OWA (IOWA) operator extends the OWA operator [41]. Considering the importance of arguments, the Importance IOWA (I-IOWA) operator was defined in [5]. Driven by the I-IOWA operator, we associate the importance of $s_{l}$ with its possibility degree for HFLTSs and define the new importance-guided possibility distribution generation method with linguistic quantifier.

Definition 7: "Let $S=\left\{s_{0}, s_{1}, \ldots, s_{g}\right\}$ be a linguistic term set and $H_{S}=\left\{s_{L}, s_{L+1}, \ldots, s_{U}\right\}$ be a HFLTS given by an expert with $0 \leq L \leq U \leq g$. The importance-guided possibility distribution for $H_{S}$ on $S$ generated by means of a RIM linguistic quantifier $Q$ is represented by $P=\left\{p_{0}, \ldots, p_{l}, \ldots, p_{g}\right\}$, where $p_{l}$ is given as

$$
p_{l}=\left\{\begin{array}{c}
0, \quad l=0,1, \ldots, L-1 \\
Q\left(\frac{T(l-L+1)}{T(U-L+1)}\right)-Q\left(\frac{T(l-L)}{T(U-L+1)}\right), \quad l=L, \ldots, U \\
0, \quad l=U+1, \ldots, g
\end{array}\right.
$$

$T(U-L+1)=\sum_{j=1}^{U-L+1} f_{\sigma(j)}, \quad T(l-L+1)=\sum_{j=1}^{l-L+1} f_{\sigma(j)}$, and $f_{\sigma(j)}$ is an importance function where $\sigma:\{1,2, \ldots, U-L+1\} \rightarrow\{1,2, \ldots, U-L+1\}$ is a permutation function such that $\sigma(j+1) \leq \sigma(j)$ for $j \in\{L, L+$ $1, \ldots, U-1\} . "$

Example 4: Considering the average linguistic quantifier of Example 1, the importance-guided possibility values when $l=L, L+1, \cdots, U$ are calculated as follows:

$$
p_{l}=Q\left(\frac{T(l-L+1)}{T(U-L+1)}\right)-Q\left(\frac{T(l-L)}{T(U-L+1)}\right)=\frac{\sum_{j=1}^{l-L+1} f_{\sigma(j)}}{\sum_{j=1}^{U-L+1} f_{\sigma(j)}}-\frac{\sum_{j=1}^{l-L} f_{\sigma(j)}}{\sum_{j=1}^{U-L+1} f_{\sigma(j)}}=\frac{f_{\sigma(l-L+1)}}{\sum_{j=1}^{U-L+1} f_{\sigma(j)}}
$$

If $f_{\sigma(j)}$ represents the probability density function of the exponential distribution, then equation (10) coincides with the method developed by Chen et al. [4] given in definition 3.

From the conclusions drawn from the provided examples, it is proved that the existent possibility distribution generation methods are actually special cases of the proposed linguistic quantifier based possibility distribution generation methods for HFLTSs, which justify its validity. Furthermore, the proposed method allows as well the implementation of the fuzzy majority concept, which has not been considered before in the existing possibility distribution generation methods. 
To perform comparison of and computation with HFLTSs, the quantifier-based expected linguistic term of HFLTSs, which is denoted as $E\left(H_{S}\right)$, is introduced

$$
E\left(H_{S}\right)=\Delta\left(\sum_{l=0}^{g} \Delta^{-1}\left(s_{l}, 0\right) p_{l}\right)=\Delta\left(\sum_{l=L}^{U} \Delta^{-1}\left(s_{l}, 0\right)\left(Q\left(\frac{U-l+1}{U-L+1}\right)-Q\left(\frac{U-l}{U-L+1}\right)\right)\right)
$$

where $\Delta$ and $\Delta^{-1}$ are the one to one mappings of the linguistic 2-tuple representation model [13].

Example 5: Let $H_{S}=\left\{s_{2}, s_{3}, s_{4}, s_{5}\right\}$ be a HFLTS on $S=\left\{s_{0}, s_{1}, \ldots, s_{6}\right\}$. Using the possibility distribution with the linguistic quantifier 'most' of Example 2, $P=\{0,0,0.1,0.5,0.4,0,0\}$, the quantifier-based expected linguistic term of $H_{S}$ is $E\left(H_{S}\right)=\left(s_{3}, 0.3\right)$.

Summarizing, in this section the following three main research contributions have been obtained:

1. A novel possibility distribution generation method with linguistic quantifier for an HFLTS is defined.

2. The new possibility distribution generation method generalizes the two main existing methods in the literature reported in [4,31].

3. The new proposed method is also useful to implement the concept of soft majority in the resolution process of the decision making problem.

\section{Attitude linguistic quantifier based possibility distribution generation method for HFLTSs}

In group decision problems, experts might have different opinions about a linguistic quantifier due to different attitudes, which can affect its type and its parameters. Take for example the linguistic quantifier 'most' as per equation (7); one expert may believe $Q(x)=1$ when $x \geq 0.8$, while another may consider this to be when $x \geq 0.9$. In such cases, we should adjust the membership function of the linguistic quantifier to the attitudes of the experts. In fact, we could associate the attitude of experts with $Q$ to reflect the different preference for a linguistic quantifier in group decision making. The attitudes of experts to linguistic quantifier are classified into two main categories: tolerant attitude and strict attitude. Experts with tolerant attitude always assign a lower value in the unit interval with a higher membership degree. On the contrary, experts with strict attitude usually correlate higher values in the unit interval with lower membership degrees. The new attitude linguistic quantifier concept is therefore introduced and defined as follows.

Definition 8: "Let $\gamma,-1<\gamma<1$, be the attitude index of experts and $Q$ a RIM linguistic quantifier. The following attitude linguistic quantifier is introduced

$$
Q_{a t t}(x)=\left\{\begin{array}{cc}
Q\left(\min \left\{\frac{x}{\frac{1-\gamma}{1+\gamma}}, 1\right\}\right), & 0 \leq x<1 \\
1, & x=1
\end{array}\right.
$$

If $0 \leq \gamma<1, Q_{a t t}$ represents a tolerant attitude linguistic quantifier; while if $-1<\gamma \leq 0$, $Q_{\text {att }}$ represents a strict attitude linguistic quantifier."

Experts can choose a suitable $\gamma$ value to express their attitudes towards an existing linguistic quantifier $Q$. When $\gamma$ tends to -1 or 1 , the corresponding attitude to the linguistic quantifier is strengthening. Specifically, the closer $\gamma$ is to 1 , the more tolerant an expert is. On the contrary, the closer $\gamma$ is to -1 , the stricter the expert is. Finally, when $\gamma$ tends to 0 , the corresponding attitude to the linguistic quantifier is lessening, with $\gamma$ equals to 0 indicating that the expert has a neutral attitude to the existing quantifier; indeed, in this case $Q$ is not affected by the expert attitude because $Q_{\text {att }}$ coincides with $Q$.

The proposed attitude linguistic quantifier is associated to a classic linguistic quantifier. The attitude index is included in the proposed quantifier to reflect the preference of experts for the quantifier, which make the new quantifier more suitable for its application in decision making with multiple experts having different preferences. Although the proposed attitude linguistic quantifier is based on an original linguistic quantifier modified via an alterable attitude index, it still retains the same properties of the classic linguistic quantifier, i.e the attitude linguistic 
quantifier is also a RIM quantifier. This is proved in the following result.

Theorem 1: The attitude linguistic quantifier $Q_{a t t}$ is a RIM quantifier.

Proof: We need to prove that properties 1-3 of definition 4 are verified by $Q_{a t t}$.

1) $Q_{\text {att }}(0)=Q(0)=0$.

2) According to definition $8, Q_{a t t}(1)=1$.

3) $\frac{x}{\frac{1-\gamma}{1+\gamma}}$ can be re-written as $\frac{x}{\frac{2}{1+\gamma}-1}$. On the one hand, for $-1<\gamma \leq 0$, it is clear that $\frac{x}{\frac{2}{1+\gamma}-1} \in(0, x]$. Because $x \leq$ 1 , then $Q_{a t t}$ can be expressed as

$$
Q_{a t t}(x)=\left\{\begin{array}{cc}
Q\left(\frac{x}{\frac{2}{1+\gamma}-1}\right), & 0 \leq x<1 \\
1, & x=1
\end{array}, \text { if }-1<\gamma \leq 0 .\right.
$$

We discuss the monotonicity of $Q_{a t t}$ with $\gamma$ fixed.

- If $1>x>y$ then $\frac{x}{\frac{2}{1+\gamma}-1}>\frac{y}{\frac{2}{1+\gamma}-1}$ The monotonicity of $Q$ implies that $Q\left(\frac{x}{\frac{2}{1+\gamma}-1}\right) \geq Q\left(\frac{y}{\frac{2}{1+\gamma}-1}\right)$ and consequently $Q_{a t t}(x) \geq Q_{a t t}(y)$.

- If $1=x>y$, then we have that $Q(x)=Q_{a t t}(x)=1$ and because $Q$ is a RIM quantifier it is $1 \geq$ $Q\left(\frac{y}{\frac{2}{1+\gamma}-1}\right)$, then $Q_{a t t}(x) \geq Q_{a t t}(y)$.

On the other hand, for $0 \leq \gamma<1$ it is $\frac{x}{\frac{2}{1+\gamma}-1} \in[x, \infty)$. We will discuss the two possible scenarios:

- If $\frac{x}{\frac{2}{1+\gamma}-1} \in[x, 1)$, then $Q_{a t t}(x)=\left\{\begin{array}{cc}Q\left(\frac{x}{\frac{2}{1+\gamma}-1}\right), & 0 \leq x<1 \\ 1, & x=1\end{array}\right.$ For a fixed $\gamma$, it was proved before that $Q_{a t t}(x) \geq Q_{a t t}(y)$ if $x>y$.

- If $\frac{x}{\frac{2}{1+\gamma}-1} \in[1, \infty)$, then $Q_{a t t}(x)=Q(1)=1$ and because $Q$ is a RIM quantifier it means that $Q_{a t t}(x) \geq$ $Q_{a t t}(y)$ if $x>y$.

In conclusion, it is true that $Q_{a t t}(x) \geq Q_{a t t}(y)$ whenever $x>y$.

Therefore, $Q_{a t t}$ verifies properties 1-3 of definition 4 and it is a RIM quantifier.

As shown in Theorem 1, the attitude linguistic quantifier, which synthesizes the attitude index and RIM linguistic quantifier, has preserved the basic properties of RIM linguistic quantifier. Considering the tolerant attitude and the strict attitude represent the opposite preference on linguistic quantifier, the following theorem will present the way in which the attitude indices impact the direction of the original quantifier.

Theorem 2: If $0 \leq \gamma<1, Q_{a t t}(x) \geq Q(x) \forall x$, while if $-1<\gamma \leq 0, Q_{a t t}(x) \leq Q(x) \forall x$.

Proof: 1) When $0 \leq \gamma<1$, from Theorem 1 it is $Q_{a t t}(x)=Q\left(\frac{x}{\frac{2}{1+\gamma}-1}\right)$ for $0 \leq x<1$ when $\frac{x}{\frac{2}{1+\gamma}-1} \in[x, 1)$ and $Q_{a t t}(x)=Q(1)=1$ for $0 \leq x<1$ when $\frac{x}{\frac{2}{1+\gamma}-1} \geq 1$. According to the non-decreasing property of RIM linguistic quantifiers, it is $Q_{a t t}(x)=Q\left(\frac{x}{\frac{2}{1+\gamma}-1}\right) \geq Q(x)$ when $\frac{x}{\frac{2}{1+\gamma}-1}<1$; and $Q_{a t t}(x)=Q(1) \geq Q(x)$ when $\frac{x}{\frac{2}{1+\gamma}-1} \geq 1$. Thus, $Q_{a t t}(x) \geq Q(x)$ for $0 \leq x<1$, which in conjunction with $Q_{a t t}(1)=Q(1)$ imply that if $0 \leq \gamma<1$ then $Q_{\text {att }}(x) \geq Q(x) \forall x$. 
2) For $-1<\gamma \leq 0$, from Theorem 1 it is $Q_{a t t}(x)=\left\{\begin{array}{cc}Q\left(\frac{x}{\frac{2}{1+\gamma}-1}\right), & 0 \leq x<1 \\ 1, & x=1\end{array}\right.$. In this case, it is $\frac{x}{\frac{2}{1+\gamma}-1} \in(0, x]$ and non-decreasing property of RIM linguistic quantifiers implies that $Q_{a t t}(x)=Q\left(\frac{x}{\frac{2}{1+\gamma}-1}\right) \leq Q(x)$ for $0 \leq x<$ 1 , which in conjunction with $Q_{a t t}(1)=Q(1)$ imply that if $-1<\gamma \leq 0, Q_{a t t}(x) \leq Q(x) \forall x$.

As it is well known, there are two special types of RIM quantifiers: 'for all ' $\left(Q_{*}\right)$ and 'there exist' $\left(Q^{*}\right)$, which are defined, respectively, as follows [34]:

$$
\begin{aligned}
& Q_{*}(1)=1 \text { and } Q_{*}(x)=0 \forall x \neq 1 \\
& Q^{*}(0)=0 \text { and } Q^{*}(x)=1 \forall x \neq 0
\end{aligned}
$$

Yager in [38] proposed an important result showing that all RIM quantifiers are bounded by $Q_{*}$ and $Q^{*}$. The following theorem demonstrates that the proposed attitude linguistic quantifier possesses a similar property. Indeed, the following results states that $Q_{a t t}$ is equivalent to the quantifier 'there exist' when $\gamma \rightarrow 1$, while it is equivalent to the quantifier 'for all' when $\gamma \rightarrow-1$.

Theorem 3: For $\gamma \rightarrow 1$ it is $Q_{a t t}(x) \rightarrow Q^{*}(x)$, while for $\gamma \rightarrow-1$ it is $Q_{a t t}(x) \rightarrow Q_{*}(x)$.

Proof: From definition 8, we have

$$
-1<\gamma<1 \Rightarrow Q_{a t t}(x)=\left\{\begin{array}{cc}
Q\left(\min \left\{\frac{x}{1-\gamma}, 1\right\}\right), & 0 \leq x<1 \\
1, & x=1
\end{array}\right.
$$

In particular, it is $Q_{a t t}(0)=Q(0)=0$ and $Q_{a t t}(1)=Q(1)=1$.

For $x \in(0,1)$, when $\gamma \rightarrow 1$ it is $\frac{1-\gamma}{1+\gamma} \rightarrow 0$ and

$$
Q\left(\min \left\{\frac{x}{\frac{1-\gamma}{1+\gamma}}, 1\right\}\right) \rightarrow Q(1)=1
$$

Thus, $\forall x: Q_{a t t}(x) \rightarrow Q^{*}(x)$ when when $\gamma \rightarrow 1$.

For $x \in(0,1)$, when $\gamma \rightarrow-1$ it is $\frac{1-\gamma}{1+\gamma} \rightarrow \infty$ and

Thus, $\forall x: Q_{a t t}(x) \rightarrow Q_{*}(x)$ when when $\gamma \rightarrow-1$.

$$
Q\left(\min \left\{\frac{x}{\frac{1-\gamma}{1+\gamma}}, 1\right\}\right) \rightarrow Q(0)=0
$$

Equation (12) and definition 8 are used to develop the corresponding attitude quantifier-guided expected linguistic term of HFLTSs:

$$
E_{\text {att }}\left(H_{S}\right)=\Delta\left(\sum_{l=L}^{U} \Delta^{-1}\left(s_{l}, 0\right)\left(Q_{a t t}\left(\frac{U-l+1}{U-L+1}\right)-Q_{a t t}\left(\frac{U-l}{U-L+1}\right)\right)\right)
$$

Since $\left\{\Delta^{-1}\left(s_{l}, 0\right) \mid l=L, \ldots, U\right\} \quad$ is equivalent to $\{L, \ldots, U\}, E_{\text {att }}\left(H_{S}\right)$ can be re-written as $E_{\text {att }}\left(H_{S}\right)=$ $\Delta\left(L+\sum_{l=1}^{U-L} Q_{a t t}\left(\frac{l}{U-L+1}\right)\right)$. With definition 8 , we have

$$
E_{\text {att }}\left(H_{S}\right)=\Delta\left(L+\sum_{l=1}^{U-L} Q\left(\min \left\{\frac{l}{\frac{(U-L+1)(1-\gamma)}{1+\gamma}}, 1\right\}\right)\right),-1<\gamma<1
$$

Apparently, $E_{a t t}\left(H_{S}\right)$ is a function of $\gamma$ and should be better denoted as $E_{a t t}\left(H_{S}(\gamma)\right)$, which can be simply expressed as $E_{\text {att }}(\gamma)$.

Considering that $E_{a t t}(\gamma)$ reflects the degree of hesitant fuzzy linguistic assessment and $\gamma$ denotes the attitude of an expert, exploring the relationship between $E_{a t t}(\gamma)$ and $\gamma$ is helpful to pursue an effective and meaningful feedback mechanism in group consensus. This is given in the following result. 
Theorem 4: The attitude quantifier-guided expected linguistic term of HFLTSs $E_{a t t}(\gamma)$ is a non-decreasing function of the attitude index $\gamma(-1,1)$.

Proof: If $\gamma_{1}>\gamma_{2}$, then $\frac{1-\gamma_{1}}{1+\gamma_{1}}<\frac{1-\gamma_{2}}{1+\gamma_{2}}$ and $\frac{l}{\frac{(U-L+1)\left(1-\gamma_{1}\right)}{1+\gamma_{1}}}>\frac{l}{\frac{(U-L+1)\left(1-\gamma_{2}\right)}{1+\gamma_{2}}}$. There are three possible cases:

1) $\frac{l}{\frac{(U-L+1)\left(1-\gamma_{1}\right)}{1+\gamma_{1}}}>\frac{l}{\frac{(U-L+1)\left(1-\gamma_{2}\right)}{1+\gamma_{2}}} \geq 1$. In this case, it is:

$$
E_{a t t}\left(\gamma_{1}\right)=E_{a t t}\left(\gamma_{2}\right)=\Delta\left(L+\sum_{l=1}^{U-L} Q(1)\right)=\Delta(L+U-L)=\Delta(U)=s_{U} .
$$

2) $\frac{l}{\frac{(U-L+1)\left(1-\gamma_{1}\right)}{1+\gamma_{1}}} \geq 1>\frac{l}{\frac{(U-L+1)\left(1-\gamma_{2}\right)}{1+\gamma_{2}}}$. In this case, it is:

$$
E_{a t t}\left(\gamma_{1}\right)=\Delta\left(L+\sum_{l=1}^{U-L} Q(1)\right)=s_{U} \text { and } E_{a t t}\left(\gamma_{2}\right)=\Delta\left(L+\sum_{l=1}^{U-L} Q\left(\frac{l}{\frac{(U-L+1)\left(1-\gamma_{2}\right)}{1+\gamma_{2}}}\right)\right) .
$$

According to the non-decreasing property of $Q$, we have $E_{a t t}\left(\gamma_{1}\right) \geq E_{a t t}\left(\gamma_{2}\right)$.

3) $1>\frac{l}{\frac{(U-L+1)\left(1-\gamma_{1}\right)}{1+\gamma_{1}}}>\frac{l}{\frac{(U-L+1)\left(1-\gamma_{2}\right)}{1+\gamma_{2}}}$. In this case, it is:

$$
E_{a t t}\left(\gamma_{1}\right)=\Delta\left(L+\sum_{l=1}^{U-L} Q\left(\frac{l}{\frac{(U-L+1)\left(1-\gamma_{1}\right)}{1+\gamma_{1}}}\right)\right) \text { and } E_{a t t}\left(\gamma_{2}\right)=\Delta\left(L+\sum_{l=1}^{U-L} Q\left(\frac{l}{\frac{(U-L+1)\left(1-\gamma_{2}\right)}{1+\gamma_{2}}}\right)\right) .
$$

Again, according to the non-decreasing property $Q$, we have $E_{a t t}\left(\gamma_{1}\right) \geq E_{a t t}\left(\gamma_{2}\right)$.

Summarizing, if $\gamma_{1}>\gamma_{2}$, then $E_{a t t}\left(\gamma_{1}\right) \geq E_{a t t}\left(\gamma_{2}\right)$, i.e. $E_{a t t}(\gamma) E_{\text {att }}(\gamma)$ is a non-decreasing function of $\gamma$.

In this section the following three main research contributions have been achieved:

1. An attitude linguistic quantifier is defined to reflect the preference of experts for linguistic quantifier in the form of attitude index. This quantifier fulfills the basic properties of RIM linguistic quantifiers.

2. The effect of the attitude index on the direction of change of the attitude linguistic quantifier with respect to the original quantifier is provided.

3. As a result of managing an HFLTS, the expected linguistic term of HFLTSs with attitude linguistic quantifier is defined and proved to be the non-decreasing function with respect to the attitude parameter, which is an important characteristic that is exploited in proposing the following new consensus reaching process in multi-criteria GDM problems with HFLTSs.

\section{Consensus reaching process in multi-criteria GDM problems with HFLTSs}

In a multi-criteria group decision making problem in a HFLTS framework consists we have $t$ experts, $\Xi=$ $\left\{d^{1}, d^{2}, \ldots, d^{t}\right\}$, who assess $n$ alternatives, $A=\left\{A_{1}, A_{2}, \ldots, A_{n}\right\}$, with respect to $m$ criteria, $C=\left\{C_{1}, C_{2}, \ldots, C_{m}\right\}$. The assessment on alternative $i$ with respect to criterion $j$ provided by expert $d^{l}$ is represented by $r_{i j}^{l}$, which is a HFLTS with respect to the linguistic term set $S=\left\{s_{0}, s_{1}, \ldots, s_{g}\right\}$. In this setting, we have an assessment matrix $R^{l}=$ $\left(r_{i j}^{l}\right), i=1, \ldots, n ; j=1, \ldots, m ; l=1, \ldots, t$. To rank the alternatives, the collective assessment matrix is derived using suitable aggregated methods. If there are some experts whose assessments are rather different to the assessments of other members in the group, the aggregated collective assessment would not be effective and it might not be accepted by the group as its 'true representation'. Therefore, a consensus model should be carried out in advance to the collective preference aggregation stage being activated.

Inspired by the methods introduced by Herrera-Viedma et al. [14] and $\mathrm{Wu}$ and $\mathrm{Xu}$ [31], an appropriate consensus framework that integrates the attitude index into the feedback mechanism to guarantee the convergence of group consensus is developed next.

\subsection{Consensus measurement}

Three levels of consensus measurement are introduced: 
LEVEL 1: Similarity of pairs (alternatives, criteria). To capture the degree of agreement between two experts $d^{l}$ and $d^{k}$, the following similarity index with embedded attitude is defined

$$
I_{i j}^{l k}=1-\frac{1}{g}\left|\Delta^{-1}\left(E_{a t t}\left(r_{i j}^{l}\right)\right)-\Delta^{-1}\left(E_{a t t}\left(r_{i j}^{k}\right)\right)\right|
$$

where $i=1, \ldots, n ; j=1, \ldots, m ; l, k=1, \ldots, t$ and $E_{a t t}\left(r_{i j}^{*}\right)$, the expected linguistic term of alternative $i$ with respect to criterion $j$ for expert* ${ }^{*}$, is computed using equation (20). The HFLTS $r_{i j}^{l}$ is a subset of the linguistic term set $S=$ $\left\{s_{0}, s_{1}, \ldots, s_{g}\right\}$, and therefore it is $0 \leq \Delta^{-1}\left(E_{a t t}\left(r_{i j}^{*}\right)\right) \leq g$. As a result, $I_{i j}^{l k}$ is in the closed unit interval $[0,1]$. The average similarity degree between expert $d^{l}$ and the rest of experts $d^{k}, k \neq l$, is interpreted as the similarity index of $d^{l}$,

$$
I_{i j}^{l}=\frac{1}{t-1} \sum_{k=1, k \neq l}^{t} I_{i j}^{l k}, i=1, \ldots, n ; j=1, \ldots, m ; l=1, \ldots, t
$$

Therefore, $I_{i j}^{l} \in[0,1]$ measures the consensus level of expert $d^{l}$ with respect to pairs of alternatives and criteria.

LEVEL 2: Similarity of alternatives. The similarity degree of alternatives by an expert $d^{l}$ is computed as

$$
I_{i}^{l}=\sum_{j=1}^{m} w_{j} I_{i j}^{l}, i=1, \ldots, n ; l=1, \ldots, t
$$

where $w_{j}, j=1, \ldots, m$ denotes the weights associated to the set of criteria . In the absence of additional information on this, the criteria weights can be considered all equal, that is $w_{j}=\frac{1}{m}, j=1, \ldots, m$. This is the assumption in the rest of the paper. The similarity degree of alternatives represents the consensus level of experts $d^{l}$ with respect to alternatives assessment.

LEVEL 3: Similarity of experts. The general similarity degree for an expert $d^{l}$ is computed as

$$
I^{l}=\frac{1}{n} \sum_{i=1}^{n} I_{i}^{l}=\frac{1}{m n} \sum_{i=1}^{n} \sum_{j=1}^{m} I_{i j}^{l}=\frac{1}{m n(t-1)} \sum_{k=1, k \neq l}^{t} \sum_{i=1}^{n} \sum_{j=1}^{m} I_{i j}^{l k}
$$

$I^{l} \in[0,1]$ indicates the consensus degree of the expert $d^{l}$. It can be used to identify the experts contributing less to group consensus.

\subsection{Identification process}

According to the above consensus measurement methodology, consensus can be evaluated at different levels, which can be useful, when group consensus is not sufficient, to identify which experts, in which alternatives and for which criteria, are contributing less to consensus. To achieve this, the below three processes are applied:

1) Experts identification. The set of experts with consensus level lower than a group acceptable threshold $\theta$ is:

$$
D(l)=\left\{d^{l} \mid I^{l}<\theta, l=1, \ldots, t\right\}
$$

2) Alternatives identification. The set of alternatives which can be adjusted by experts in $D(l)$ are:

$$
A(i)=\left\{A_{i} \mid I_{i}^{l}<\theta \wedge d^{l} \in D(l)\right\}
$$

3) Criteria identification. The set of criteria with respect to alternatives in $A(i)$ that require adjustment are:

$$
A C(i, j)=\left\{(i, j) \mid I_{i j}^{l}<\theta \wedge A_{i} \in A(i)\right\}
$$

Experts might reject to change their evaluations on the identified alternatives and criteria if the proposed changes lead to a substantially different modified assessment to the original one. If experts accept only the change of one criterion for each alternative evaluation in each round, then $A C(i, j)$ could be replaced with

$$
A C\left(i, j_{\text {min }}\right)=\left\{\left(i, j_{\text {min }}\right) \mid I_{i j_{\text {min }}}^{l}=\min _{j}\left\{I_{i j}^{l}, j=1, \ldots, m\right\} \wedge A_{i} \in A(i)\right\}
$$

With the identification sets $D(l), A(i)$ and $A C(i, j)$, the position of the value which may be adjusted to achieve higher consensus level is identified as follows: 


$$
P O S=\left\{r_{i j}^{l} \mid l \in D(l),(i, j) \in A C(i, j)\right\}
$$

\subsection{Consensus reaching process}

The consensus threshold is a crucial parameter in the process of reaching group consensus. In the existing literature, the threshold value is determined using one of the following two main approaches: subjective experience method and simulation analysis method. For the former, the threshold value is given according to the subjective experience of the set of experts and the decision-making problem [6,14,30,31]. For the latter, a simulation analysis software such as MATLAB is utilized to analyze the relationship between the threshold and some other related objects of the problem, such as the number of iterations [33] or the number of alternatives [24]. In the present study, the first method is used and the threshold value is assumed to be agreed on by the experts in advance to execute the consensus process, and therefore it is assumed to be given. For the purpose of illustration, in the examples provided within the rest of the paper this has been assumed to be set at a relatively high 0.8 value.

The group consensus threshold $\theta$ is achieved when all experts' consensus levels are above it, i.e. when the following condition $I^{l} \geq \theta, l=1, \ldots, t$ is satisfied. Thus, if there are some experts whose consensus levels are lower than the threshold value $\theta$. i.e. $\exists i, I^{l}<\theta$, then these experts will be identified in the experts identification step described above, and if they are to increase their consensus level they will have to adjust their assessments on some of the alternatives identified in the subsequent alternatives identification step as described above.

There are two possible routes to modify assessments. On the one hand, the identified experts can revise the evaluation value in the identified criteria with respect to the identified alternatives in the identified position. On the other hand, they can modify their attitudes to the linguistic quantifier. The later operation would affect the entire elements of the matrix and would be an effective method for reaching the consensus threshold value. Indeed, according to Theorem 4, the attitude expected linguistic value of $r_{i j}^{l}, E_{a t t}\left(r_{i j}^{l}\right)$, will increase if the attitude index $\gamma$ increases. As $E_{a t t}\left(r_{i j}^{l}\right)$ reflect the preference of experts to pairs of alternatives and criteria, the modification of the attitude index can affect every evaluation value of $R^{l}$ and then the consensus degree of the group.

Thus, the approach proposed here synthesizes the two above adjustment approaches to develop a novel feedback mechanism for achieving a satisfied consensus, which is to be conducted in two stages: attitude adjustment and assessment adjustment. Attitude adjustment will change all of the elements in the assessment matrix and it will cause rapid adjustments of evaluation and a quick increase of the consensus level. Nevertheless, sometimes experts are not willing to change their attitudes or only accept the adjustment to a certain value. For example, an expert who prefers a strict attitude will be willing to accept a change of his attitude index from -0.8 to -0.4 , but an adjustment of his attitude index from -0.8 to 0.4 , i.e. a change that would make him to move from a strict to a tolerant attitude, which could be considered a too brusque change of attitude, might not be justifiable and not acceptable to the expert. In that case though, to improve his/her consensus level, the expert would be receptive to assessment adjustment of those identified values from the criteria identification step in order.

The mathematical formalization of the proposed two stages feedback mechanism of attitude adjustment and assessment adjustment is elaborated below:

Stage 1: Attitude adjustment. Let us recall equation (24), $I_{i j}^{l k}=1-\frac{1}{g}\left|\Delta^{-1}\left(E_{a t t}\left(r_{i j}^{l}\right)\right)-\Delta^{-1}\left(E_{a t t}\left(r_{i j}^{k}\right)\right)\right|$, which measures the similarity of pairs of alternatives and criteria assessments given by two experts. To begin with, the influence of increasing the value of the attitude index is analyzed. In this case, each expected value of elements in the matrix $E_{a t t}\left(r_{i j}^{l}\right)$ will increase accordingly. However, in terms of equation (24), it is not sure that an increase of $E_{a t t}\left(r_{i j}^{l}\right)$ will lead to a decrease or an increase of $I_{i j}^{l k}$.

To help analyzing this issue, $I_{i j}^{l k}$ is split into two parts to analyze the results. For simplicity, $E_{i j}^{l}$ is used as equivalent to $\Delta^{-1}\left(E_{a t t}\left(r_{i j}^{l}\right)\right)$. Then $I_{i j}^{l k}$ is re-written as $I_{i j}^{l k}=1-\frac{1}{g}\left|E_{i j}^{l}-E_{i j}^{k}\right|$. Let $I_{i j}^{l k+}=1-\frac{1}{g}\left(E_{i j}^{l}-E_{i j}^{k}\right)$, if $E_{i j}^{l}>E_{i j}^{k}$ and $I_{i j}^{l k-}=1-\frac{1}{g}\left(E_{i j}^{k}-E_{i j}^{l}\right)$, if $E_{i j}^{l} \leq E_{i j}^{k}$. Let us assume now that $E_{i j}^{l}$ changes to $E_{i j}^{l}+\delta^{l}$ where $\delta^{l}$ might be either positive or negative, then $I_{i j}^{l k+}(+), I_{i j}^{l k-}(+)$ and $I^{l}(+)$ are introduced to represent the situation of 
the results obtained with $E_{i j}^{l}+\delta^{l}$.

$$
\begin{aligned}
& I_{i j}^{l k+}(+)=1-\frac{1}{g}\left(E_{i j}^{l}+\delta^{l}-E_{i j}^{k}\right)=I_{i j}^{l k+}-\frac{1}{g} \delta^{l} \\
& I_{i j}^{l k-}(+)=1-\frac{1}{g}\left(E_{i j}^{k}-\left(E_{i j}^{l}+\delta^{l}\right)\right)=I_{i j}^{l k-}-\frac{1}{g}\left(-\delta^{l}\right) .
\end{aligned}
$$

Then, according to equation (27) it is

$$
\begin{aligned}
& I^{l}(+)=\frac{1}{m n(t-1)} \sum_{k=1, k \neq l}^{t} \sum_{i=1}^{n} \sum_{j=1}^{m} I_{i j}^{l k}(+)
\end{aligned}
$$

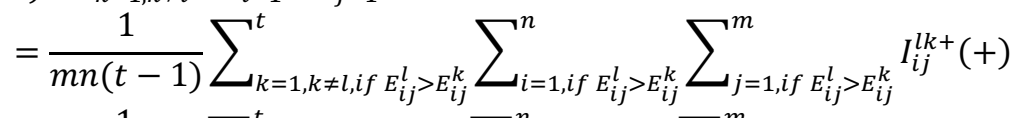

$$
\begin{aligned}
& +\frac{1}{m n(t-1)} \sum_{k=1, k \neq l, i f E_{i j}^{l} \leq E_{i j}^{k}}^{t} \sum_{i=1, i f E_{i j}^{l} \leq E_{i j}^{k}}^{n} \sum_{j=1, i f E_{i j}^{l} \leq E_{i j}^{k}}^{m} I_{i j}^{l k-}(+) \\
& =\frac{1}{m n(t-1)} \sum_{k=1, k \neq l, i f E_{i j}^{l}>E_{i j}^{k}}^{t} \sum_{i=1, i f E_{i j}^{l}>E_{i j}^{k}}^{n} \sum_{j=1, i f E_{i j}^{l}>E_{i j}^{k}}^{m}\left(I_{i j}^{l k+}-\frac{1}{g} \delta^{l}\right) \\
& +\frac{1}{m n(t-1)} \sum_{k=1, k \neq l, i f E_{i j}^{l} \leq E_{i j}^{k}}^{t} \sum_{i=1, i f E_{i j}^{l} \leq E_{i j}^{k}}^{n} \sum_{j=1, i f E_{i j}^{l} \leq E_{i j}^{k}}^{m}\left(I_{i j}^{l k-}-\frac{1}{g}\left(-\delta^{l}\right)\right)
\end{aligned}
$$

Let $\quad N H^{l}=\left\{E_{i j}^{k} \mid \# H\left(r_{i j}^{l}\right)>1 \wedge E_{i j}^{l}>E_{i j}^{k}, k \neq l ; i=1, \ldots, n ; j=1, \ldots, m\right\} \quad$ and $\quad N L^{l}=$ $\left\{E_{i j}^{k} \mid \# H\left(r_{i j}^{l}\right)>1 \wedge E_{i j}^{l} \leq E_{i j}^{k}, k \neq l ; i=1, \ldots, n ; j=1, \ldots, m\right\}$, where \#H( $\left.r_{i j}^{l}\right)$ is the cardinality of the HFLTS with

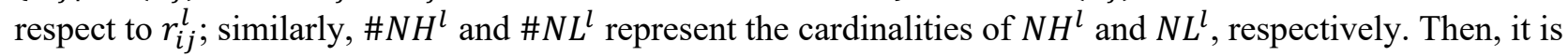

$$
I^{l}(+)=\frac{1}{m n(t-1)} \sum_{k=1, k \neq l}^{t} \sum_{i=1}^{n} \sum_{j=1}^{m} I_{i j}^{l k}-\frac{\delta^{l}}{m n(t-1) g}\left(\# N H^{l}-\# N L^{l}\right)=I^{l}-\frac{\delta^{l}}{m n(t-1) g}\left(\# N H^{l}-\# N L^{l}\right)
$$

In other words, $I^{l}(+)-I^{l}=-\frac{\delta^{l}}{m n(t-1) g}\left(\# N H^{l}-\# N L^{l}\right)$. When $I^{l}(+)-I^{l}>0$, which indicates an increase of consensus, then we have $\delta^{l}\left(\# N H^{l}-\# N L^{l}\right)<0$. That is to say the increase of consensus depends on $\delta^{l}$ and the differences between \#NH $H^{l}$ and \#NL $L^{l}$. As $\delta^{l}$ represents the change of $E_{i j}^{l}$ which is affected by the attitude indices $\gamma^{l}$, we can determine the direction of change of the attitude indices $\gamma^{l}$ according to the difference between \#NH $H^{l}$ and \#NL $L^{l}$. Specifically, if \#NH $H^{l}-\# N L^{l}>0$, then $\# N H^{l}-\# N L^{l}$ will be obtained by reducing the attitudes index because this will make $E_{i j}^{l}$ decrease, i.e. it will make $\delta^{l}<0$, and vice versa. Consequently, the identified experts, $d^{l} \in D(l)$, will be provided with the following feedback rules for changing their attitude indices:

- $\quad$ ATT-R1: If \#NH $H^{l}-\# N L^{l}<0$, expert $d^{l}$ should increase their attitude indices $\gamma^{l}$.

- ATT-R2: If \#NH $H^{l}-\# N L^{l}>0$, expert $d^{l}$ should decrease their attitude indices $\gamma^{l}$.

- ATT-R3: If \#NH $H^{l}-\# N L^{l}=0$, then expert $d^{l}$ do not have to change their attitude indices $\gamma^{l}$.

We have the following results that validate the effectiveness of the proposed attitudinal feedback process:

Proposition 2: Feedback rules ATT-R1, ATT-R2 and ATT-R3 imply that $I^{l}(+)-I^{l}>0$ and the group consensus will increase.

Proposition 3: Because the consensus level as per (27) is bounded, the proposed set of feedback rules ATT-R1, $A T T-R 2$ and ATT-R3 for adjusting the attitude indices guarantees the convergence of the consensus reaching process.

Nevertheless, it can happen that the consensus levels for some experts are possibly still lower than the threshold value after adjusting their attitude indices to values they can compromise at the greatest extent. That may be caused by the great deviation on certain alternatives and criteria between individual and collective evaluations. Therefore the modification in the following stage focuses on the value of alternatives and criteria where consensus level is still lower than the threshold value after Stage 1. 
Due to the adjustment in the Stage 1 feedback process, the expected linguistic values of the elements of the decision matrix of experts $d^{l} \in D(l)$ will have changed. Then the similarity indices need to be calculated again, and the process in section 5.2 is to be implemented again to obtain the new identification sets after Stage 1: $D^{1}(l)$ and $A C^{1}(i, j)$. Following this, we can determine the set of position, $P O S=\left\{r_{i j}^{l} \mid l \in D^{1}(l),(i, j) \in A C^{1}(i, j)\right\}$, which should be adjusted according to the feedback rules in Stage 2 below.

Stage 2 : Assessment adjustment. In this case, the determination of the direction of change requires the collective group evaluation to be obtained in advance. Let $E_{a t t}\left(r_{i j}^{c}\right)$ be the collective expected value of alternative $A_{i}$ with respect to criterion $C_{j}$ which is satisfied by a majority of experts based on a linguistic quantifier OWA aggregation:

$$
E_{a t t}\left(r_{i j}^{c}\right)=\Delta\left(\sum_{l=1}^{t} \lambda^{l} \Delta^{-1}\left(E_{a t t}\left(r_{i j}^{b(l)}\right)\right)\right)
$$

where the OWA weights $\lambda^{l}$ satisfy $0<\lambda^{l}<1, \sum_{l=1}^{t} \lambda^{l}=1$; and $b(l)$ is the decreasing permutation of $l$ with respect to the importance degree associated to the experts. In the absence of information regarding importance degrees of experts, all experts are considered equally important and the OWA weights are computed using Yager's approach $\lambda^{l}=Q\left(\frac{l}{t}\right)-Q\left(\frac{l-1}{t}\right)$; however, if experts have different importance degrees, the impotence OWA weights are computed using the expression $\lambda^{l}=Q\left(\frac{S_{l}}{T}\right)-Q\left(\frac{S_{l-1}}{t}\right)$, where $T=\sum_{k=1}^{t} f_{k}, S=\sum_{k=1}^{l} f_{k}$ and $f_{k}$ is the importance of expert $k$. Based on $E_{a t t}\left(r_{i j}^{c}\right)$, the feedback rules for changing the evaluation of alternative with respect to criteria are:

- ASS-R1: If $E_{a t t}\left(r_{i j}^{l}\right)<E_{a t t}\left(r_{i j}^{c}\right)$, then expert $d^{l}$ should increase the value of $r_{i j}^{l}$.

- ASS-R2: If $E_{a t t}\left(r_{i j}^{l}\right)>E_{a t t}\left(r_{i j}^{c}\right)$, then expert $d^{l}$ should decrease the value of $r_{i j}^{l}$.

- ASS-R3: If $E_{a t t}\left(r_{i j}^{l}\right)=E_{a t t}\left(r_{i j}^{c}\right)$, then expert $d^{l}$ don not need to change the value of $r_{i j}^{l}$.

Similarly to the feedback process Stage 1, we have the following results that validate the effectiveness of the proposed evaluation feedback process:

Proposition 4: The proposed set of feedback rules ASS-R1, ASS-R2 and ASS-R3 for adjusting the evaluation of alternatives with respect to criteria reduces the deviation between the individual and collective evaluation values in the identified position, and the consensus level of the group will increase accordingly.

Proposition 5: Because the consensus level as per (27) is bounded, the proposed set of feedback rules ASS-R1, ASS$R 2$ and $A S S-R 3$ for adjusting the evaluation of alternatives with respect to criteria guarantees the convergence of the consensus reaching process.

When the decision evaluation reach the group consensus threshold, the collective group evaluation obtained through an aggregation process is satisfactory and acceptable for the whole group and the ranking of alternatives in terms of the collective group evaluation with equation (36) can be derived. Under the previous assumption that all criteria have equal weights, the overall performance of alternatives can be defined as follows

$$
E_{a t t}\left(r_{i}^{c}\right)=\Delta\left(\frac{1}{m} \sum_{j=1}^{m} \Delta^{-1}\left(E_{a t t}\left(r_{i j}^{c}\right)\right)\right)
$$

Consequently, as $E_{a t t}\left(r_{i}^{c}\right)$ is a 2-tuple linguistic variable, the ranking of alternatives can be derived by applying the comparison method of the linguistic 2-tuple representation model [13].

Summarizing, in this section, a new two stages feedback mechanism of attitude adjustment and assessment adjustment is devised to guarantee the convergence of the consensus reaching process. The attitude adjustment can change all of the elements in the assessment matrix, which will cause rapid adjustments of evaluation and a quick increase of the consensus level. 


\section{Proposed multiple criteria group decision making framework with HFLTSs information}

Group decision making is a process in which a group of experts give their preference on a set of alternatives and interact to achieve consensus on the final chosen alternative solution. Consensus level is a crucial state of agreement for the group, which leads to the degree of satisfaction of the decision making result. The consensus process can be implemented through several experts interaction rounds in which feedback rules are applied and information derived from them are returned to experts to help them accelerate the group consensus achievement. The present research study concerns both experts' preference assessments and attitude in the process of managing HFLTS information, thus the proposed consensus process incorporate feedback rules for both preference assessments and attitude indices.

In this section, the new proposed group decision making framework with HFLTS information is described. Firstly, the HFLTS information is transformed to possibility distribution of linguistic terms with attitude linguistic quantifier and the expected linguistic value of HFLTSs are computed (Phase I). Then, the consensus reaching process is carried out until a satisfactory consensus level is achieved (Phase II). Finally, the selection process will be implemented to produce a ranking of alternatives (Phase III). Specifically, the algorithmic process of group decision making with HFLTS information is provided in next section, followed by an illustrative example of its application.

\subsection{The process of multiple criteria group decision making with HFLTS information}

The group decision making with HFLTS information framework consists of three phases:

\section{Phase I: HFLTS information managing process}

Step 1: Deriving the possibility distribution of alternatives with respect to criteria as per the HFLTSs and linguistic quantifier and attitude indices provided by experts (definition 8 followed by definition 6).

Step 2: Calculating the expected linguistic term of each element in the decision matrices via equation (20).

\section{Phase II: Consensus reaching process}

Step 3: Calculating the three levels of similarity using equations (24)-(27) to measure consensus levels for experts, alternatives and pairs of alternatives and criteria.

Step 4: Determining whether the group consensus level achieves the group consensus threshold. If the threshold consensus level is achieved, then move to Phase III; else, the 'Identification process' equations (28)-(30) are carried out.

Step 5: Applying Stage 1: Attitude adjustment feedback rules ATT-R1, ATT-R2 and ATT-R3 to generate the new possibility distribution and expected linguistic term for the HFLTSs matrices.

Step 6: Calculating the three levels of similarity using equation (24)-(27) to measure updated consensus level of identified experts. Determining whether the group consensus after the attitude adjustment feedback process has reached the group consensus threshold. If the threshold consensus level is achieved, then move to Phase III; else, the $P O S$ set is obtained using equation (32).

Step 7: Applying Stage 2: Assessment adjustment feedback rules ASS-R1, ASS-R2 and ASS-R3 to generate the new decision matrix. Go back to Step 1.

\section{Phase III: Selection Process}

Step 8: Computing the collective group evaluation and overall performance of alternatives using equations (36) and (37), respectively, and derive the final ranking of alternatives using the comparison method of the linguistic 2tuple representation model [13].

The group decision making with HFLTS information framework is depicted in Fig. 3. 


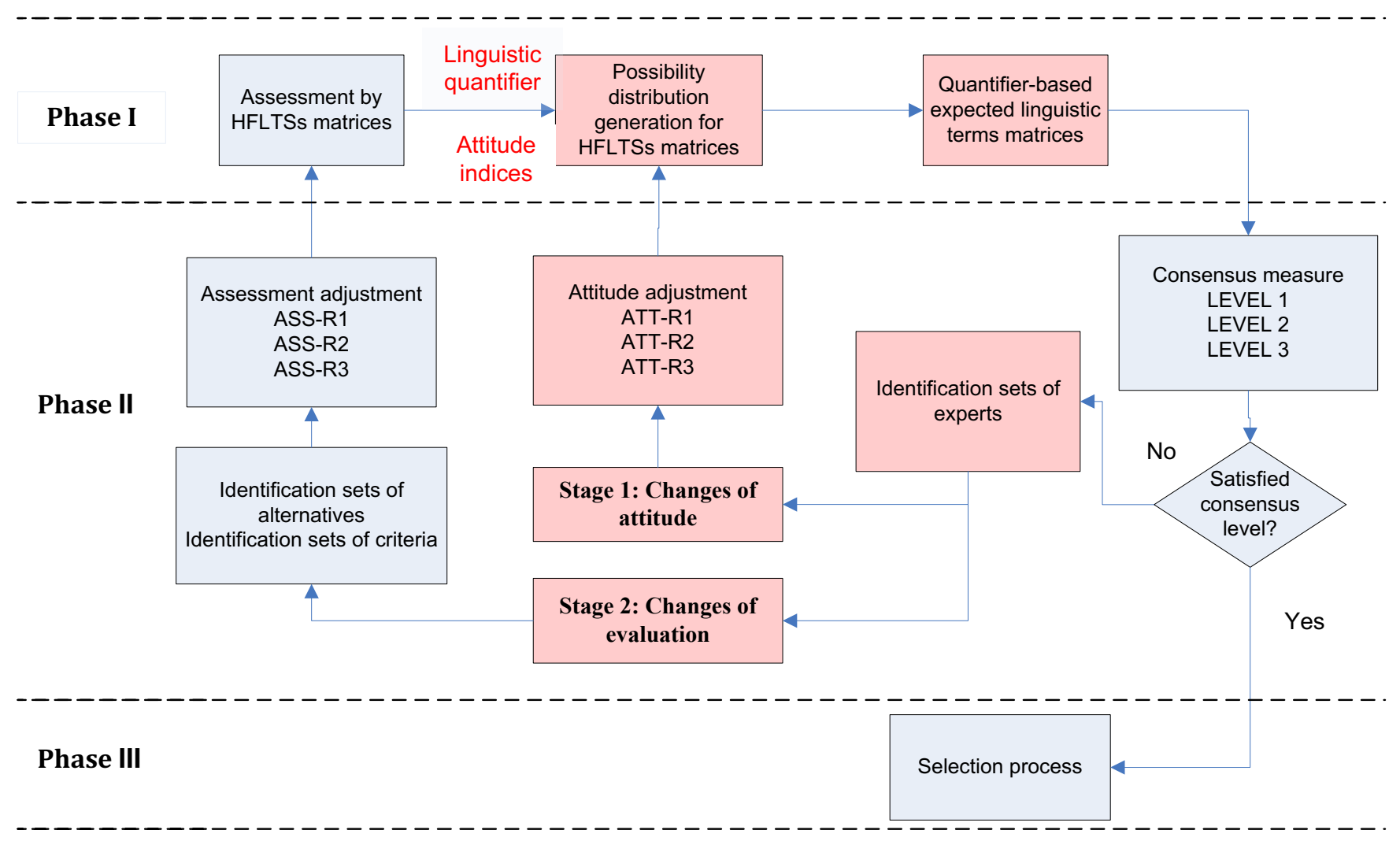

Fig. 3. The proposed framework of multiple criteria group decision making with HFLTSs information (main contributions highlighted in red)

\subsection{Illustrative example}

In this section, we use the example provided in [31] to numerically illustrate the proposed framework of multiple criteria group decision making problems with HFLTS information. The example scenario describes a manufacturing company that evaluates four candidates, $A=\left\{A_{1}, A_{2}, A_{3}, A_{4}\right\}$, for a vacant position. Four experts, $\Xi=$ $\left\{d^{1}, d^{2}, d^{3}, d^{4}\right\}$, are elected to assess the four candidates with respect to six criteria, $C=\left\{C_{1}, C_{2}, \ldots, C_{6}\right\}$, using the following linguistic term set:

$$
S=\left\{s_{0}=\text { None, } s_{1}=\text { Very Low }, s_{2}=\text { Low }, s_{3}=\text { Medium, } s_{4}=\text { High, } s_{5}=\text { Very High, } s_{6}=\text { Perfect }\right\} \text {. }
$$

Experts' HFLTSs based on $S$ to describe their assessment on the candidates according to the set of criteria can be found in [31]. In the following, the proposed framework of multiple criteria group decision making problems with HFLTS information is applied to rank the four candidates.

Phase I: HFLTS information managing process

Step 1: Deriving the possibility distribution of alternatives with respect to criteria as per the HFLTSs and linguistic quantifier and attitude indices provided by experts.

Firstly, experts choose the type of linguistic quantifier $Q$ to use and provide individual attitude to the quantifier. It is assumed herein that experts choose the quantifier 'most' to express their preference of HFLTS. That is to say that most of the linguistic terms in HFLTS satisfy the preference of the experts. Experts $d^{l}$ are assumed to provide the following different attitudes on $Q: \gamma^{1}=0, \gamma^{2}=0.2, \gamma^{3}=-0.2, \gamma^{4}=0.6$. From definition 8 and the membership function of the RIM quantifier most given in equation (7), it is: 


$$
\begin{aligned}
& Q_{a t t}\left(x_{1}\right)=Q\left(x_{1}\right) ; \quad Q_{a t t}\left(x_{2}\right)=\left\{\begin{array}{c}
0,0 \leq x_{2}<0.2 \\
3 x_{2}-0.6,0.2 \leq x_{2} \leq 0.53 ; \\
1, \quad 0.53<x_{2} \leq 1
\end{array}\right. \\
& Q_{a t t}\left(x_{3}\right)=\left\{\begin{array}{cl}
0, \quad 0 \leq x_{3}<0.45 \\
\frac{4 x_{3}}{3}-0.6, \quad 0.45 \leq x_{3}<1 ; \\
1, \quad x_{3}=1
\end{array} \quad Q_{a t t}\left(x_{4}\right)=\left\{\begin{array}{c}
0, \quad 0 \leq x_{4}<0.075 \\
8 x_{4}-0.6, \quad 0.075 \leq x_{4} \leq 0.2 \\
1, \quad 0.2<x_{4} \leq 1
\end{array}\right.\right.
\end{aligned}
$$

With definition 6 , the possibility distribution of all alternatives with respect to the criteria as per the provided HFLTSs can be given. To illustrate how the proposed method works, we provide $r_{11}^{l}, l=1,2,3,4$ as an example.

- With $r_{11}^{1}=\left\{s_{5}, s_{6}\right\}$, the possibility distribution of $r_{11}^{1}$ on $S$ is given as $P_{11}^{1}=\{0,0,0,0,0,0.6,0.4\}$.

- With $r_{11}^{2}=\left\{s_{3}, s_{4}\right\}$, the possibility distribution of $r_{11}^{2}$ on $S$ is given as $P_{11}^{2}=\{0,0,0,0.1,0.9,0,0\}$.

- With $r_{11}^{3}=\left\{s_{2}, s_{3}, s_{4}\right\}$, the possibility distribution of $r_{11}^{3}$ on $S$ is given as $P_{11}^{3}=\{0,0,0.71,0.29,0,0,0\}$.

- With $r_{11}^{4}=\left\{s_{2}, s_{3}\right\}$, the possibility distribution of $r_{11}^{4}$ on $S$ is given as $P_{11}^{4}=\{0,0,0,1,0,0,0\}$.

Similarly, we can derive the possibility distributions of the rest of alternatives with respect the criteria as per the provided HFLTSs.

Step 2: Calculating the expected linguistic term of each element in the decision matrices based on the linguistic 2-tuple representation model [13] and equation (20). The matrices with expected linguistic terms are:

$$
\begin{gathered}
E R^{1}=\left[\begin{array}{lllllll}
\left(s_{5}, 0.4\right) & \left(s_{5}, 0.4\right) & \left(s_{6}, 0\right) & \left(s_{2}, 0.4\right) & \left(s_{3},-0.2\right) & \left(s_{5}, 0.4\right) \\
\left(s_{4}, 0.4\right) & \left(s_{5}, 0.4\right) & \left(s_{5}, 0\right) & \left(s_{5}, 0.4\right) & \left(s_{4}, 0.4\right) & \left(s_{4}, 0.4\right) \\
\left(s_{3}, 0.4\right) & \left(s_{4}, 0.4\right) & \left(s_{3}, 0.4\right) & \left(s_{4}, 0.4\right) & \left(s_{5}, 0.4\right) & \left(s_{5}, 0.4\right) \\
\left(s_{5}, 0.4\right) & \left(s_{1}, 0.4\right) & \left(s_{4},-0.2\right) & \left(s_{6}, 0\right) & \left(s_{5},-0.2\right) & \left(s_{4}, 0\right)
\end{array}\right], E R^{2}=\left[\begin{array}{ccccccc}
\left(s_{4},-0.1\right) & \left(s_{5}, 0\right) & \left(s_{6},-0.1\right) & \left(s_{5},-0.1\right) & \left(s_{4},-0.1\right) & \left(s_{4},-0.1\right) \\
\left(s_{5}, 0.39\right) & \left(s_{5}, 0\right) & \left(s_{4}, 0\right) & \left(s_{6},-0.1\right) & \left(s_{5},-0.1\right) & \left(s_{4}, 0\right) \\
\left(s_{2},-0.1\right) & \left(s_{6}, 0\right) & \left(s_{5},-0.1\right) & \left(s_{4},-0.1\right) & \left(s_{6},-0.1\right) & \left(s_{4}, 0\right) \\
\left(s_{6},-0.1\right) & \left(s_{5},-0.1\right) & \left(s_{4},-0.1\right) & \left(s_{4}, 0\right) & \left(s_{6},-0.1\right) & \left(s_{5}, 0\right)
\end{array}\right], \\
E R^{3}=\left[\begin{array}{ccccccc}
\left(s_{2}, 0.293\right) & \left(s_{3}, 0.067\right) & \left(s_{4}, 0.067\right) & \left(s_{4}, 0.067\right) & \left(s_{5}, 0.067\right) & \left(s_{2}, 0.067\right) \\
\left(s_{5}, 0.067\right) & \left(s_{4}, 0.067\right) & \left(s_{4}, 0\right) & \left(s_{5}, 0.067\right) & \left(s_{4}, 0.067\right) & \left(s_{4}, 0.067\right) \\
\left(s_{4}, 0\right) & \left(s_{5}, 0.067\right) & \left(s_{4}, 0\right) & \left(s_{1}, 0.067\right) & \left(s_{5}, 0.067\right) & \left(s_{3}, 0\right) \\
\left(s_{3}, 0.067\right) & \left(s_{4}, 0.067\right) & \left(s_{2}, 0.293\right) & \left(s_{3}, 0\right) & \left(s_{5}, 0.067\right) & \left(s_{5}, 0.067\right)
\end{array}\right], E R^{4}=\left[\begin{array}{cccccc}
\left(s_{3}, 0\right) & \left(s_{4}, 0\right) & \left(s_{6}, 0\right) & \left(s_{4}, 0\right) & \left(s_{3}, 0\right) & \left(s_{4}, 0\right) \\
\left(s_{5}, 0\right) & \left(s_{6}, 0\right) & \left(s_{4}, 0\right) & \left(s_{6}, 0\right) & \left(s_{4}, 0\right) & \left(s_{5}, 0\right) \\
\left(s_{2}, 0\right) & \left(s_{6}, 0\right) & \left(s_{6}, 0\right) & \left(s_{6}, 0\right) & \left(s_{4}, 0\right) & \left(s_{5}, 0\right) \\
\left(s_{4}, 0\right) & \left(s_{5}, 0\right) & \left(s_{4}, 0\right) & \left(s_{5}, 0\right) & \left(s_{6}, 0\right) & \left(s_{3}, 0\right)
\end{array}\right] .
\end{gathered}
$$

Phase II: Consensus reaching process

Step 3: Calculating the three levels of similarity using equations (24)-(27) to measure the consensus levels for experts, alternatives and pairs of alternatives and criteria.

The similarity matrices of pairs of alternatives and criteria are

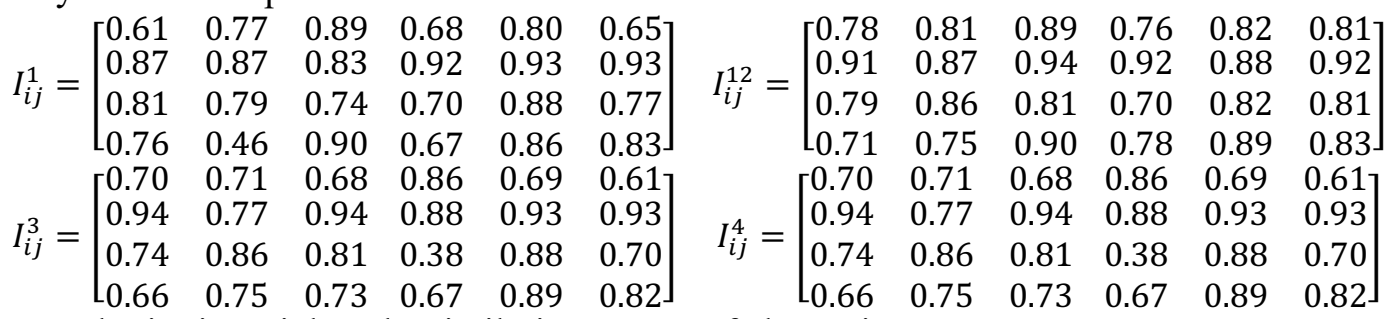

Assuming equal criteria weights, the similarity vectors of alternatives are

$$
I_{i}^{1}=\left[\begin{array}{l}
0.73 \\
0.89 \\
0.78 \\
0.75
\end{array}\right], I_{i}^{2}=\left[\begin{array}{l}
0.81 \\
0.91 \\
0.80 \\
0.81
\end{array}\right], I_{i}^{3}=\left[\begin{array}{c}
0.71 \\
0.90 \\
0.73 \\
0.75
\end{array}\right], I_{i}^{4}=\left[\begin{array}{c}
0.83 \\
0.90 \\
0.74 \\
0.79
\end{array}\right] \text {. }
$$

Finally, the similarity of experts are:

$$
I^{1}=0.79, I^{2}=0.83, I^{3}=0.77, I^{4}=0.82 .
$$

Step 4: For the purposed of comparing the results of the proposed method and those reported in [31], the consensus threshold value is set as the one used in [31], i.e. $\theta=0.8$. The following experts are identified as contributing less to consensus: 


$$
D(l)=\left\{d^{1}, d^{3}\right\}
$$

Step 5: (Stage 1: Attitude adjustment) The first and third experts should change their attitude values. Finding $\# N H$ and $\# N L$ to find out the direction of adjustment.

As $\# N H^{1}-\# N L^{1}=28-32=-4<0$ and $\# N H^{3}-\# N L^{3}=16-41=-25<0$, both experts should increase their respective attitude index values. Assuming that $\gamma^{1}$ is increased from 0 to 0.2 and $\gamma^{3}$ is increased from -0.2 to -0.1 , the new similarity matrices of pairs of alternatives and criteria are

$$
\begin{aligned}
I_{i j}^{1} & =\left[\begin{array}{llllll}
0.54 & 0.70 & 090 & 0.76 & 0.85 & 0.58 \\
0.95 & 0.85 & 0.83 & 0.96 & 0.91 & 0.91 \\
0.78 & 0.86 & 0.82 & 0.68 & 0.86 & 0.68 \\
0.75 & 0.87 & 0.85 & 0.67 & 0.93 & 0.82
\end{array}\right] \quad I_{i j}^{12}=\left[\begin{array}{lllllll}
0.76 & 0.80 & 0.90 & 0.80 & 0.85 & 0.79 \\
0.94 & 0.85 & 0.94 & 0.96 & 0.91 & 0.88 \\
0.77 & 0.90 & 0.83 & 0.68 & 0.86 & 0.78 \\
0.75 & 0.90 & 0.89 & 0.78 & 0.93 & 0.82
\end{array}\right] \\
I_{i j}^{3} & =\left[\begin{array}{llllll}
0.71 & 0.71 & 0.71 & 0.88 & 0.70 & 0.60 \\
0.96 & 0.76 & 0.94 & 0.88 & 0.91 & 0.91 \\
0.77 & 0.90 & 0.83 & 0.38 & 0.86 & 0.67 \\
0.66 & 0.90 & 0.73 & 0.67 & 0.91 & 0.80
\end{array}\right] \quad I_{i j}^{4}=\left[\begin{array}{llllll}
0.76 & 0.80 & 0.90 & 0.88 & 0.81 & 0.79 \\
0.96 & 0.84 & 0.94 & 0.95 & 0.89 & 0.90 \\
0.78 & 0.90 & 0.71 & 0.56 & 0.72 & 0.78 \\
0.75 & 0.89 & 0.89 & 0.78 & 0.92 & 0.71
\end{array}\right]
\end{aligned}
$$

The new similarity vectors of alternatives are

$$
I_{i}^{1}=\left[\begin{array}{l}
0.72 \\
0.90 \\
0.78 \\
0.81
\end{array}\right], I_{i}^{2}=\left[\begin{array}{l}
0.82 \\
0.91 \\
0.80 \\
0.84
\end{array}\right], I_{i}^{3}=\left[\begin{array}{l}
0.72 \\
0.89 \\
0.73 \\
0.78
\end{array}\right], I_{i}^{4}=\left[\begin{array}{c}
0.82 \\
0.91 \\
0.74 \\
0.82
\end{array}\right] .
$$

The new similarity of experts are:

$$
I^{1}=0.80, I^{2}=0.84, I^{3}=0.78, I^{4}=0.82
$$

The new similarity values of experts are higher than their previous ones (in Step 3), which shows that the adjustment of attitudes indices increases the group consensus (Proposition 2).

Step 6: Given the threshold $\theta=0.8$, the following sets of experts, alternatives and criteria are identified:

$$
\begin{gathered}
D^{1}(l)=\left\{d^{l} \mid I^{l}<0.8, l=1,2,3,4\right\}=\left\{d^{3}\right\} \\
A^{1}(i)=\left\{A_{i} \mid I_{i}^{l}<0.8 \wedge d^{l} \in D(l)\right\}=\left\{A_{1}, A_{3}, A_{4}\right\} \\
A C^{1}\left(i, j_{\text {min }}\right)=\left\{\left(i, j_{\text {min }}\right) \mid I_{i j_{\text {min }}}^{l}=\min _{j}\left\{I_{i j}^{l}, j=1, \ldots, 6\right\} \wedge A_{i} \in A(i)\right\}=\{(1,6),(3,4),(4,1)\}
\end{gathered}
$$

With sets $D^{1}(l), A^{1}(i)$ and $A C^{1}\left(i, j_{\min }\right)$, the following positions of alternatives and criteria for adjusting evaluations are identified:

$$
P O S=\left\{r_{16}^{3}, r_{34}^{3}, r_{41}^{3}\right\}
$$

Step 7: (Stage 2: Assessment adjustment) Obtaining the direction of changes in terms of collective group evaluation. Assuming all experts are equally important, with equation (36) and the linguistic quantifier ' $m o s t$ ', the collective group evaluation is computed as

$$
E R^{c}=\left[\begin{array}{llllll}
\left(s_{3}, 0.3096\right) & \left(s_{4}, 0.3218\right) & \left(s_{6},-0.2282\right) & \left(s_{4},-0.0227\right) & \left(s_{3}, 0.5550\right) & \left(s_{4},-0.2282\right) \\
\left(s_{5}, 0.0773\right) & \left(s_{5}, 0.2818\right) & \left(s_{4}, 0\right) & \left(s_{6},-0.1682\right) & \left(s_{4}, 0.4691\right) & \left(s_{4}, 0.4691\right) \\
\left(s_{3},-0.25\right) & \left(s_{5}, 0.4991\right) & \left(s_{4}, 0.35\right) & \left(s_{4}, 0.0318\right) & \left(s_{5}, 0.3691\right) & \left(s_{4}, 0.3\right) \\
\left(s_{5},-0.3182\right) & \left(s_{4}, 0.2591\right) & \left(s_{4},-0.2004\right) & \left(s_{4}, 0.3\right) & \left(s_{6},-0.4232\right) & \left(s_{4}, 0.3\right)
\end{array}\right] .
$$

According to Step 2, we get 


$$
E R^{3}=\left[\begin{array}{cccccc}
\left(s_{2}, 0.293\right) & \left(s_{3}, 0.067\right) & \left(s_{4}, 0.067\right) & \left(s_{4}, 0.067\right) & \left(s_{5}, 0.067\right) & \left(s_{2}, 0.067\right) \\
\left(s_{5}, 0.067\right) & \left(s_{4}, 0.067\right) & \left(s_{4}, 0\right) & \left(s_{5}, 0.067\right) & \left(s_{4}, 0.067\right) & \left(s_{4}, 0.067\right) \\
\left(s_{4}, 0\right) & \left(s_{5}, 0.067\right) & \left(s_{4}, 0\right) & \left(s_{1}, 0.067\right) & \left(s_{5}, 0.067\right) & \left(s_{3}, 0\right) \\
\left(s_{3}, 0.067\right) & \left(s_{4}, 0.067\right) & \left(s_{2}, 0.293\right) & \left(s_{3}, 0\right) & \left(s_{5}, 0.067\right) & \left(s_{5}, 0.067\right)
\end{array}\right] .
$$

Comparing the elements of $E R^{3}$ and $E R^{c}$ allows to get the following direction of change: increase $r_{16}^{3}$, increase $r_{34}^{3}$ and increase $r_{41}^{3}$. Assuming the following new evaluations $r_{16}^{3}=\left\{s_{3}, s_{4}\right\}, r_{34}^{3}=\left\{s_{2}, s_{3}\right\}, r_{41}^{3}=$ $\left\{s_{4}, s_{5}\right\}$, new individual decision matrices are built and Step 1-4 are carried out again, which would result in the following new similarity indices of experts

$$
I^{1}=0.81, I^{2}=0.85, I^{3}=0.80, I^{4}=0.85 \text {. }
$$

These values corroborate that the adjustment of assessments increases the group consensus (Proposition 4). As all of these are above the threshold value of 0.8 , it is concluded that the reached group consensus level is satisfactory, and the selection process can be activated.

Phase III: Selection Process

Step 8: Assuming that all criteria are equally important, the overall performance of alternatives are computed applying equation (37) to the collective group evaluation of Step 7.

$$
E_{\text {att }}\left(r_{1}^{c}\right)=\left(s_{4}, 0.13\right), E_{\text {att }}\left(r_{2}^{c}\right)=\left(s_{5},-0.15\right), E_{\text {att }}\left(r_{3}^{c}\right)=\left(s_{4}, 0.4\right), E_{\text {att }}\left(r_{4}^{c}\right)=\left(s_{5},-0.45\right) .
$$

According to the comparison method of the linguistic 2-tuple representation model [13], the ranking of alternatives candidates for the vacant position is:

$$
A_{2}>A_{4}>A_{3}>A_{1} \text {. }
$$

\subsection{Comparison with Wu and Xu's method in [31]}

\begin{tabular}{|c|c|c|c|c|c|}
\hline & \multirow{2}{*}{\multicolumn{2}{|c|}{ Possibility distribution generation method }} & \multicolumn{2}{|c|}{ Consensus reaching process } & \multirow{2}{*}{$\begin{array}{l}\text { Ranking of } \\
\text { alternatives }\end{array}$} \\
\hline & & & Consensus model & $\begin{array}{l}\text { Identification sets } \\
\text { and adjustment }\end{array}$ & \\
\hline \multirow{2}{*}{$\begin{array}{c}\text { Wu and } X u \\
\text { methodology } \\
{[31]}\end{array}$} & \multicolumn{2}{|c|}{$\begin{array}{l}\text { Features of the method: Elements in HFLTS are } \\
\text { equally important }\end{array}$} & \multirow{2}{*}{$\begin{array}{l}\text { - Consensus measure for } \\
\text { the level of alternatives } \\
\text { - Adjustment rules for } \\
\text { assessments }\end{array}$} & \multirow{2}{*}{$\begin{array}{l}r_{11}^{1}(-), r_{16}^{1}(-) \\
r_{15}^{3}(-), r_{31}^{3}(-) \\
r_{34}^{3}(+), r_{36}^{3}(+) \\
r_{31}^{4}(+)\end{array}$} & \multirow[b]{2}{*}{$A_{2}>A_{3}>A_{4}>A_{1}$} \\
\hline & $\begin{array}{l}\text { Take } \\
r_{11}^{3}=\left\{s_{2}, s_{3}, s_{4}\right\} \\
\text { as an example }\end{array}$ & $P_{11}^{3}=\left\{0,0, \frac{1}{3}, \frac{1}{3}, \frac{1}{3}, 0,0\right\}$ & & & \\
\hline \multirow[t]{2}{*}{$\begin{array}{c}\text { Proposed } \\
\text { methodology }\end{array}$} & \multicolumn{2}{|c|}{$\begin{array}{l}\text { Features of the method: Generating possibility } \\
\text { degree with attitude linguistic quantifier, which } \\
\text { allow for different importance values of } \\
\text { elements in HFLTS }\end{array}$} & \multirow{2}{*}{$\begin{array}{l}\text { - Consensus measure for } \\
\text { the level of experts } \\
\text { - Adjustment rules for } \\
\text { attitude indices and } \\
\text { assessments }\end{array}$} & \multirow[t]{2}{*}{$\begin{array}{l}\text { Stage 1: } \\
\quad d^{1}(+), d^{3}(+) \\
\text { Stage } 2: \\
r_{16}^{3}(+), r_{34}^{3}(+), r_{41}^{3}(+)\end{array}$} & \multirow{2}{*}{$A_{2}>A_{4}>A_{3}>A_{1}$} \\
\hline & $\begin{array}{l}\text { Take } \\
r_{11}^{3}=\left\{s_{2}, s_{3}, s_{4}\right\} \\
\text { as an example }\end{array}$ & $\begin{array}{l}P_{11}^{3} \\
=\{0,0,0.7067,0.2933,0,0,0\}\end{array}$ & & & \\
\hline
\end{tabular}

To verify the effectiveness and embody the advantages of the proposed methodology, Table 1 presents a comparison with the methodology proposed by $\mathrm{Wu}$ and $\mathrm{Xu}$ in [31] regarding the possibility distribution generation method, the consensus reaching process and the ranking of alternatives.

(1) Regarding the possibility distribution generating process, our proposed methodology considers the individual differences of experts' preferences on the possibility value in HFLTSs via the elicitation of the possibility distribution by means of their attitude associated to a linguistic quantifier. Taking $r_{11}^{3}=\left\{s_{2}, s_{3}, s_{4}\right\}$ as an example in Table 1, our proposed method produces the possibility distribution $\{0,0,0.7067,0.2933,0,0,0\}$ for the assumed third expert's attitude index of -0.2. The main difference between our proposed attitude linguistic quantifier based possibility distribution generation method for HFLTS and $\mathrm{Wu}$ and Xu's method is that our method allows for 
modeling heterogeneous importance for elements in HFLTS. In fact, as it was shown in Example 1 of Section 3, the proposed attitude linguistic quantifier based possibility distribution generation method for HFLTS extends Wu and Xu's method, which is the particular case of our proposed method when using a neutral attitude value of 0 and the average linguistic quantifier $Q(x)=x$. It is worth reminding again that our proposed methodology also extends the method developed by Chen et al. in [4]. Thus, it can be concluded that our proposed methodology allows for the implementation of additional information on experts' importance and preferences as well as their individual, and possibly different, attitudinal values, which makes it more flexible and general in its applicability in real decision making problems than the two existing methods mentioned.

(2) Our proposed methodology utilizes the consensus measure method to elicit the similarity of each expert rather than the similarity of each alternative approach utilized by the methodology of $\mathrm{Wu}$ and $\mathrm{Xu}$ [31]. Also, the attitude index of experts is considered in a new feedback mechanism to adjust their whole assessment quickly. As shown in Table 1, our proposed adjustment process is divided into two stages: attitude adjustment and assessment adjustment. Experts contributing less to consensus are identified and given feedback on the direction of change of their attitude indices in a first stage, and which assessments they should adjust in a second stage. Because Wu and Xu's methodology [31] does not concern attitude of experts, their feedback mechanism only focuses on the adjustment of assessments. In any case, there is still a difference on the amount of changes required by both approaches, with slightly lower number of direct changes required by experts in our proposed methodology (total of 5; 2 attitude changes and 3 assessment changes) than in $\mathrm{Wu}$ and $\mathrm{Xu}$ 's methodology (total of 7 assessment changes). This is because the experts' attitude index values are closely relevant to the assessment of alternatives, as described by Theorem 4. As a result, the adjustment of attitude indices leads to the quick changes (computed automatically by the proposed system, with no direct intervention by the experts required) of assessment of alternatives.

(3) Regarding the ranking of alternatives, the results obtained by our proposed methodology and the ones obtained in [31] differ. The first and the last ranked alternatives, $A_{2}$ and $A_{1}$, are the same in both cases; while the second and third ranked alternatives are swapped as a consequence of the differences explained in points (1) and (2) above. As shown in Table 1, the assessment value of $A_{3}$ is increased less in our proposed model than in the model of [31]; while the assessment value of $A_{4}$ is increased in our proposed consensus model but not changed in the model of [31]. In other words, the adjustment rules in the feedback mechanism, which is one of our contributions in this paper, truly impact the ranking of alternatives and the decision making results.

In multi-criteria group decision-making problems, the ranking of alternatives is influenced by many factors, including the criteria weights, aggregation process, experts' preference and group consensus. The assessment of the alternatives ranking method is to be made based on the reliability of the decision-making methodology applied for its derivation. In our methodology, a novel possibility distribution generating method that incorporates the linguistic quantifier and attitude preference is proposed to manage the HFLTS information, which allows more abundant possibility distribution information than previous methodologies as well as the implementation of the concept of soft majority if necessary. Our proposed group consensus reaching method consists of a two stages feedback mechanism that includes the adjustment of experts' attitude preference, which was shown to increase the efficiency of consensus achievement and to guarantee the convergence of the consensus reaching process. Consequently, when compared with existing alternatives ranking methods in the hesitant fuzzy linguistic framework, our proposed alternatives ranking method is more comprehensive as it allows to reflect the diversity of HFLTS information management, the attitude preference of experts and it is efficient in enhancing group consensus.

\subsection{Discussion}

In this section, we discuss the main differing characteristics of our proposed methods in comparison with existing research methods on this area. 
(1) Our proposed methodology incorporates the linguistic quantifier to generate possibility distribution of HFLTS for the first time. The linguistic quantifier reflects the preference of experts on HFLTSs when they assess the alternatives. In existing research, scholars have assumed that experts consider the individual linguistic terms in HFLTS to have associated equal possibility values [31], which is not an appropriate assumption because it does not cover the situation with different preference and attitude of experts. Also, the possibility distribution method based on normal distribution or exponential distribution has been proposed in [4], which put forward an assumption that underlines an in-depth knowledge background of experts to identify these distributions parameters, is in itself in contradiction with the research framework of uncertainty used to justify the provision of less precise hesitant fuzzy linguistic assessments rather than precise and exact numerical assessments. Some other studies [20] employ the concept of frequency to elicit the possibility value of linguistic terms in the group perspective, which means that all experts will end having to implement the same set of possibility values (although different) associated to the linguistic terms. In comparison with previous approaches on this topic, our proposed approach provides a novel computational approach based on the use of linguistic quantifier to express the individual preference to HFLTS, is intuitive and closer to experts' cognitive habits, does not add additional burden to experts in terms of providing precise numeric statements on probability distribution; and does not rely on other group members to obtain their individual possibility values associated to their individual HFLTS assessments. Furthermore, since there have been vast achievements about linguistic quantifier and OWA aggregation model [34,36,38], our proposed quantifier driven possibility distribution generation method has the potential to be applicable to further decision frameworks.

(2) As experts' evaluations are affected by subjective preference and cognitive styles, elements such as attitudes, experiences and knowledge background are inevitably involved in group decision problems. Attitude related decision-making approaches have been widely developed [2,26,40].. The difference with these existing approaches resides in that our approach pays attention to the attitude to linguistic quantifiers as to represent the preference of experts to HFLTSs. Considering the influence of the attitude, an attitude quantifier deriving method is developed as an important basis to generate possibility distribution in the HFLTSs framework that extends previous approaches by $\mathrm{Wu}$ and $\mathrm{Xu}$ [31] and Chen et al. [4]. Our proposed method expands the application area of linguistic quantifiers and provides a new line of research to manage HFLTSs information.

(3) Consensus reaching approach is an important issue in group decision making problems. Many scholars have developed a number of methods that include: consensus measure of various types of preference [6,7,42]; feedback mechanism [30]; optimization-based consensus model [45,46]; consensus model with preference information [49,50]; dynamic consensus process model [21] and consensus model for large scale group decision making $[15,18]$. Our proposed method incorporates the attitude character towards reaching consensus for the first time and it supports experts adjust their evaluation quickly and automatically as it is taken care of by the systems, which reduces the direct number of adjustments in consensus reaching process required from experts. Nevertheless, as attitude is related to experts' inherent preference for uncertainty, there is limitations on changes of attitude indices, which will lead to limitations on the amount of increase of the consensus level.

\section{Conclusion}

The hesitant fuzzy linguistic term set (HFLTS) is a useful tool to express hesitancy among linguistic terms in decision making. In the past few years, many approaches have contributed to the management of HTLTSs. The possibility distribution generation method of HTLTS is a powerful tool to deal with the hesitant fuzzy linguistic information. This paper presented a new possibility distribution generation method that combines the attitude linguistic quantifier and a new feedback mechanism in group decision making problems with hesitant fuzzy linguistic information. The research contributions of the present article are summarized as follows:

- A novel possibility distribution generation method with linguistic quantifier is proposed. The proposed method is consistent with the previous possibility distribution generating methods in that they become particular types of the proposed approach for specific linguistic quantifiers. Indeed, the proposed method is more general because it can lead to the uniform distribution or provides different possibility degrees for 
HFLTSs, i.e. existing methods are particular cases of the proposed one.

- An attitude linguistic quantifier has been defined, which enhances the flexibility of implementing linguistic quantifier and enlarges the range of the outcome of the proposed possibility distribution generation method.

- Considering the impact of attitude in consensus reaching process, a new two stages feedback mechanism with attitude adjustment and assessment adjustment has been developed, which guarantees the convergence to consensus. In comparison with existing literature, the process of attitude adjustment, which can change all of the elements in the assessment matrix by means of modifying the attitude index, contributes to the rapid achievement of a satisfied consensus state.

Preferences of experts for HFLTSs can be represented in a diverse range of ways which could lead to abundant HFLTSs computational models. The possibility distribution generation method for HFLTSs embedding fuzzy rather than numerical attitudes indices merits future research efforts.

\section{Acknowledgment}

This work is supported in part by the National Natural Science Foundation of China under Grant 71603242 and 71301149; the Humanity and Social Science Youth foundation of Ministry of Education under Grant 15YJC630030; the Natural Science Foundation of Zhejiang Province under Grant LY17G010002; and the China Scholarship Council (CSC) Grant 201708330087.

\section{References}

[1] H. Bin Liu, R.M. Rodríguez, A fuzzy envelope for hesitant fuzzy linguistic term set and its application to multicriteria decision making, Inf. Sci. 258 (2014) 220-238.

[2] T.-Y. Chen, An outranking approach using a risk attitudinal assignment model involving Pythagorean fuzzy information and its application to financial decision making, Appl. Soft Comput. 71 (2018) 460-487.

[3] Z.S. Chen, K.S. Chin, Y.L. Li, Y. Yang, Proportional hesitant fuzzy linguistic term set for multiple criteria group decision making, Inf. Sci. 357 (2016) 61-87.

[4] Z.S. Chen, K.S. Chin, N.Y. Mu, S.H. Xiong, J.P. Chang, Y. Yang, Generating HFLTS possibility distribution with an embedded assessing attitude, Inf. Sci. 394-395 (2017) 141-166.

[5] F. Chiclana, E. Herrera-Viedma, F. Herrera, S. Alonso, Some induced ordered weighted averaging operators and their use for solving group decision-making problems based on fuzzy preference relations, Eur. J. Oper. Res. 182 (2007) $383-399$.

[6] F. Chiclana, J.M. Tapia García, M.J. del Moral, E. Herrera-Viedma, A statistical comparative study of different similarity measures of consensus in group decision making, Inf. Sci. 221 (2013) 110-123.

[7] F. Chiclana, J.M.T. García, M.J. del Moral, E. Herrera-Viedma, Analyzing Consensus Measures in Group Decision Making, Procedia Comput. Sci. 55 (2015) 1000-1008.

[8] R. Degani, G. Bortolan, The problem of linguistic approximation in clinical decision making, Int. J. Approx. Reason. 2 (1988) $143-162$.

[9] B. Ervural, S. Ceren Oner, V. Coban, C. Kahraman, A novel Multiple Attribute Group Decision Making methodology based on Intuitionistic Fuzzy TOPSIS, in Proc. IEEE Int. Conf. Fuzzy Syst. (FUZZ-IEEE), Istanbul,Turkey, Aug. 2015, pp. 1-6 .

[10] B. Ervural, B. Ervural, O. Kabak, Evaluation of Flexible Manufacturing Systems Using a Hesitant Group Decision Making Approach, J. Intell. Syst. 2017.

[11] B.Ç. Ervural, B. Ervural, C. Kahraman, Fuzzy Sets in the Evaluation of Socio-Ecological Systems: An Interval-Valued Intuitionistic Fuzzy Multi-criteria Approach BT - Fuzzy Logic in Its 50th Year: New Developments, Directions and Challenges, in: C. Kahraman, U. Kaymak, A. Yazici (Eds.), Springer International Publishing, Cham, 2016: pp. 309-326.

[12] E. Herrera-Viedma, F.J. Cabrerizo, J. Kacprzyk, W. Pedrycz, A review of soft consensus models in a fuzzy environment, Inf. Fusion. 17 (2014) 4-13.

[13] F. Herrera, L. Martínez, A 2-tuple fuzzy linguistic representation model for computing with words, IEEE Trans. Fuzzy Syst. 8 (2000) 746-752. 
[14] E. Herrera-Viedma, L. Martínez, F. Mata, F. Chiclana, A consensus support system model for group decision-making problems with multigranular linguistic preference relations, IEEE Trans. Fuzzy Syst. 13 (2005) 644-658.

[15] Á. Labella, Y. Liu, R.M. Rodríguez, L. Martínez, Analyzing the performance of classical consensus models in large scale group decision making: A comparative study, Appl. Soft Comput. 67 (2018) 677-690.

[16] H.C. Liao, L.Y. Yang, Z.S. Xu, Two new approaches based on ELECTRE II to solve the multiple criteria decision making problems with hesitant fuzzy linguistic term sets, Appl. Soft Comput. 63 (2018) 223-234.

[17] N. Liu, Y. He, Z. Xu, A new approach to deal with consistency and consensus issues for hesitant fuzzy linguistic preference relations, Appl. Soft Comput. 76 (2019) 400-415.

[18] X. Liu, Y. Xu, F. Herrera, Consensus model for large-scale group decision making based on fuzzy preference relation with selfconfidence: Detecting and managing overconfidence behaviors, Inf. Fusion. 52 (2019) 245-256.

[19] X.W. Liu, S.L. Han, Orness and parameterized RIM quantifier aggregation with OWA operators: A summary, Int. J. Approx. Reason. 48 (2008) 77-97.

[20] Q. Pang, H. Wang, Z.S. Xu, Probabilistic linguistic term sets in multi-attribute group decision making, Inf. Sci. 369 (2016) 128143.

[21] I.J. Pérez, F.J. Cabrerizo, S. Alonso, Y.C. Dong, F. Chiclana, E. Herrera-Viedma, On dynamic consensus processes in group decision making problems, Inf. Sci. 459 (2018) 20-35.

[22] R.M. Rodriguez, L. Martinez, F. Herrera, Hesitant fuzzy linguistic term sets for decision making, IEEE Trans. Fuzzy Syst. 20 (2012) 109-119.

[23] R.M. Rodríguez, L. Martínez, F. Herrera, A group decision making model dealing with comparative linguistic expressions based on hesitant fuzzy linguistic term sets, Inf. Sci. 241 (2013) 28-42.

[24] M. Tang, X.Y. Zhou, H.C. Liao, J.P. Xu, H. Fujita, F. Herrera, Ordinal consensus measure with objective threshold for heterogeneous large-scale group decision making, Knowledge-Based Syst. 180 (2019) 62-74.

[25] V. Torra, Hesitant fuzzy sets, Int. J. Intell. Syst. 25 (2010) 529-539.

[26] S.P. Wan, F. Wang, J.Y. Dong, A novel risk attitudinal ranking method for intuitionistic fuzzy values and application to MADM, Appl. Soft Comput. J. 40 (2016) 98-112.

[27] H. Wang, Extended hesitant fuzzy linguistic term sets and their aggregation in group decision making, Int. J. Comput. Intell. Syst. 8 (2015) 14-33.

[28] J. Wang, Y.I. Lin, A fuzzy multicriteria group decision making approach to select configuration items for software development, Fuzzy Sets Syst. 134 (2003) 343-363.

[29] H. Wang, Z.S. Xu, X.-J. Zeng, Hesitant fuzzy linguistic term sets for linguistic decision making: Current developments, issues and challenges, Inf. Fusion. 43 (2018) 1-12.

[30] J. Wu, L.F. Dai, F. Chiclana, H. Fujita, E. Herrera-Viedma, A minimum adjustment cost feedback mechanism based consensus model for group decision making under social network with distributed linguistic trust, Inf. Fusion. 41 (2018) $232-242$.

[31] Z.B. Wu, J.P. Xu, Possibility Distribution-Based Approach for MAGDM with Hesitant Fuzzy Linguistic Information, IEEE Trans. Cybern. 46 (2016) 694-705.

[32] Z.S. Xu, A method based on linguistic aggregation operators for group decision making with linguistic preference relations, Inf. Sci. 166 (2004) 19-30.

[33] X.H. Xu, Z.J. Du, X.H. Chen, Consensus model for multi-criteria large-group emergency decision making considering noncooperative behaviors and minority opinions, Decis. Support Syst. 79 (2015) 150-160.

[34] R.R. Yager, Connectives and quantifiers in fuzzy sets, Fuzzy Sets Syst. 40 (1991) 39-75.

[35] R.R. Yager, A new methodology for ordinal multiobjective decisions based on fuzzy sets, in: Readings Fuzzy Sets Intell. Syst., Elsevier, 1993: pp. 751-756.

[36] R.R. Yager, Families of OWA operators, Fuzzy Sets Syst. 59 (1993) 125-148.

[37] R.R. Yager, An approach to ordinal decision making, Int. J. Approx. Reason. 12 (1995) 237-261.

[38] R.R. Yager, Quantifier guided aggregation using OWA operators, Int. J. Intell. Syst. 11 (1996) 49-73.

[39] R.R. Yager, OWA aggregation over a continuous interval argument with applications to decision making, IEEE Trans. Syst. Man, Cybern. Part B. 34 (2004) 1952-1963. 
[40] R.R. Yager, On ordered weighted averaging aggregation operators in multi-criteria decision making, in: IEEE Trans. Syst. Man,and Cybern., Elsevier, 1988: pp. 183-190.

[41] R.R. Yager, D.P. Filev, Induced ordered weighted averaging operators, IEEE Trans. Syst. Man, Cybern. Part B. 29 (1999) 141150 .

[42] H.B. Yan, T. Ma, V.N. Huynh, On qualitative multi-attribute group decision making and its consensus measure: A probability based perspective, Omega. 70 (2017) 94-117.

[43] L.A. Zadeh, The concept of a linguistic variable and its application to approximate reasoning-I, Inf. Sci. 8 (1975) 199-249.

[44] L.A. Zadeh, A computational approach to fuzzy quantifiers in natural languages, Comput. Math. with Appl. 9 (1983) 149-184.

[45] Y. Li, H.J. Zhang, Y.C. Dong, The interactive consensus reaching process with the minimum and uncertain cost in group decision making, Appl. Soft Comput. 60 (2017) 202-212.

[46] B. Zhang, H. Liang, Y. Gao, G. Zhang, The optimization-based aggregation and consensus with minimum-cost in group decision making under incomplete linguistic distribution context, Knowledge-Based Syst. 162 (2018) 92-102.

[47] G.Q. Zhang, Y.C. Dong, Y.F. Xu, Consistency and consensus measures for linguistic preference relations based on distribution assessments, Inf. Fusion. 17 (2014) 46-55.

[48] H.J. Zhang, Y.C. Dong, F. Chiclana, S. Yu, Consensus efficiency in group decision making: A comprehensive comparative study and its optimal design, Eur. J. Oper. Res. 275 (2019) 580-598.

[49] Y.X. Zhang, Z.S. Xu, H.C. Liao, A consensus process for group decision making with probabilistic linguistic preference relations, Inf. Sci. 414 (2017) 260-275.

[50] S.T. Zhang, J.J. Zhu, X.D. Liu, Y. Chen, Z.Z. Ma, Adaptive consensus model with multiplicative linguistic preferences based on fuzzy information granulation, Appl. Soft Comput. 60 (2017) 30-47. 